ACCEPTED By AJ 2004 February 5

Preprint typeset using LATEX style emulateapj v. 11/12/01

\title{
TOOLS FOR IDENTIFYING SPURIOUS LUMINOSITY OFFSETS IN TULLY-FISHER STUDIES: APPLICATION AT LOW REDSHIFT AND IMPLICATIONS FOR HIGH REDSHIFT
}

\author{
Sheila J. KANNAPPAN ${ }^{1}$ \& Elizabeth J. BARTON ${ }^{2}$ \\ Accepted by AJ 2004 February 5
}

\begin{abstract}
Studies of high-redshift galaxies usually interpret offsets from the Tully-Fisher (TF) relation as luminosity evolution. However, apparent luminosity offsets may actually reflect anomalous velocity widths. Rotation curve anomalies such as strong asymmetries or radial truncation are probably common in high- $z$ samples, due to frequent galaxy interactions and in some cases low $\mathrm{S} / \mathrm{N}$ data, although low physical resolution may mask these anomalies. In this paper we analyze well-resolved, one-dimensional optical emission-line rotation curves from two low- $z$ samples: the Close Pairs Survey, which contains a high frequency of interacting galaxies, and the Nearby Field Galaxy Survey (NFGS), which represents the general galaxy population. Unlike most low- $z$ TF samples, but in the spirit of many high- $z$ samples, these surveys reflect the natural diversity of emission-line galaxy morphologies, including peculiar, interacting, and early-type galaxies. We adopt objective, quantitative criteria to reject galaxies with severe kinematic anomalies, and we use a statistical velocity width measure that is insensitive to minor kinematic distortions. Severely anomalous galaxies are roughly twice as frequent in the Close Pairs Survey as in the NFGS, and these galaxies" TF offsets collectively resemble the "differential luminosity evolution" claimed in some high- $z$ studies, with larger offsets at lower luminosities. With the anomalous galaxies rejected, however, the TF relations for the Close Pairs Survey and the NFGS are quite similar. Furthermore, the two surveys follow very similar relations between color and TF residuals. The Close Pairs Survey color-TF residual relation extends to bluer colors and brighter TF residuals. Strong outliers from this relation are virtually always kinematically anomalous. As a result, the color-TF residual relation can serve as a powerful tool for separating reliable luminosity offsets from offsets associated with kinematic anomalies. This tool may prove especially useful at high $z$, where direct detection of kinematic distortions is not always feasible. Although we cannot reliably measure luminosity evolution for galaxies with kinematic anomalies, the TF offsets associated with these anomalies may offer a sensitive probe of evolution in the frequency and intensity of mergers and interactions on different mass scales. We perform a preliminary reanalysis of high- $z$ TF data from the FORS Deep Field and find: (1) overall luminosity evolution of $\sim 0.3 \mathrm{mag}$; (2) strong slope evolution driven by kinematically anomalous galaxies, which show TF offsets of up to $\sim 2$ mag at low luminosities; and (3) an additional zero-point offset of $\sim 0.2$ mag linked to kinematically anomalous galaxies.
\end{abstract}

Subject headings: distance scale — galaxies: evolution — galaxies: fundamental parameters — galaxies: general — galaxies: interactions — galaxies: kinematics and dynamics

\section{INTRODUCTION}

As the fundamental scaling relation between luminosity and rotation velocity for disk galaxies, the Tully-Fisher relation (TF relation, Tully \& Fisher 1977) evolves along with the galaxies that define it, reflecting general trends in mass assembly and star formation. Numerous studies have sought to trace the star formation history of disk galaxies via the redshift evolution of the zero point of the TF relation, under the assumption that TF zero point offsets represent luminosity evolution. Awkwardly, some studies find minimal luminosity evolution to redshifts as high as $z \sim 1$ (e.g., Forbes et al. 1996; Vogt et al. 1997; Bershady et al. 1999), while others report substantial 1.5-2 mag offsets at lower redshifts (e.g., Rix et al. 1997; Simard \& Pritchet 1998). Efforts to reconcile these results have generally invoked differential evolution, in which only low-luminosity galaxies evolve significantly (e.g., Simard \& Pritchet 1998; Ziegler et al. 2002).

However, some "luminosity offsets" may actually be velocity offsets. In the TF relation, underestimated rotation velocities look exactly like enhanced luminosities. The following three examples are particularly relevant to high $z$ studies.

(1) Optical emission-line data for high- $z(z \sim 0.25-1$ for this paper) blue compact galaxies may not extend to large enough radii to sample peak rotation velocities, based on $21 \mathrm{~cm} \mathrm{HI} \mathrm{stud-}$ ies of analogous galaxies at low $z$ (Barton \& van Zee 2001; Pisano et al. 2001; see also Kobulnicky \& Gebhardt 2000; Courteau \& Sohn 2003). Of course, high- $z$ studies must employ optical lines rather than HI to measure rotation velocities. Also, most high- $z$ studies have selection biases favoring the bright galaxy cores and strong emission lines typical of blue compact galaxies. One might hope that high- $z$ studies would be insensitive to radially truncated emission-line data, because unlike low$z$ analyses, high- $z$ analyses usually derive rotation velocities by analyzing kinematic and photometric profiles together (e.g., Vogt et al. 1996; Simard \& Pritchet 1999; Ziegler et al. 2002). However, such modeling techniques typically rely on simplifying assumptions that blue compact galaxies probably routinely violate, such as the assumption of a close correspondence between emission-line and underlying disk-continuum fluxes, or the assumption that rotation curves can be simply parametrised based on exponential-disk fits to the spatial flux distribution regardless of disturbances or central mass concentrations.

(2) Low S/N can also cause radially truncated emission and underestimated rotation velocities, especially in samples already biased toward galaxies with centrally concentrated emis-

${ }^{1}$ Harlan Smith Fellow, McDonald Observatory, The University of Texas at Austin, 1 University Station C1402, Austin, TX 78712-0259; sheila@ astro.as.utexas.edu

${ }^{2}$ Hubble Fellow, The University of Arizona, Steward Observatory, 933 N. Cherry Ave., Tucson, AZ 85721; ebarton@as.arizona.edu 
sion. Accounting for $\mathrm{S} / \mathrm{N}$-induced rotation curve truncation could significantly reduce discrepancies between high- $z$ TF studies (as discussed for the Simard \& Pritchet and Vogt et al. studies by Kannappan 2001).

(3) Distorted rotation curves may also yield unreliable rotation estimates and systematic velocity offsets. In low- $z$ TF samples that contain interacting or morphologically peculiar galaxies, disturbances in longslit optical emission-line rotation curves clearly correlate with apparent luminosity boosts from the TF relation (Barton et al. 2001; Kannappan et al. 2002). We suspect that these apparent boosts may not be pure luminosity offsets, especially when they are larger than would be expected based on colors or $\mathrm{H} \alpha$ equivalent widths (Kannappan et al. 2002).

At low $z$, large TF offsets associated with distorted or truncated rotation curves are most common for low-luminosity galaxies, and the affected galaxies often display emission-line S0 or irregular morphologies, sometimes with independent evidence of interactions (Kannappan et al. 2002; see also Kobulnicky \& Gebhardt 2000; Barton et al. 2001). Although most low- $z$ TF studies would reject such galaxies (e.g., Courteau 1997; Haynes et al. 1999; Tully \& Pierce 2000), all of the high- $z$ studies that report substantial faint-end luminosity evolution employ selection criteria that would admit them (e.g., Rix et al. 1997; Simard \& Pritchet 1998; Mallén-Ornelas et al. 1999; Ziegler et al. 2002). Furthermore, the frequency of such galaxies may be enhanced in high- $z$ samples to the extent that the interaction rate increases with $z$ (Patton et al. 2002; Murali et al. 2002). These points raise the obvious concern that high- $z$ TF samples may contain a population of galaxies whose velocity offsets mimic differential luminosity evolution.

Another key consideration in interpreting apparent luminosity offsets is the possibility of third-parameter dependence in TF residuals. Numerous studies have examined possible physical drivers of TF offsets, including morphology, surface brightness, gas content, environment, and color, for TF samples chosen by a variety of criteria (e.g., Roberts 1978; Rubin et al. 1985; Giraud 1986; Pierce \& Tully 1988; Mould et al. 1989; Pierce \& Tully 1992; Sprayberry et al. 1995; Courteau \& Rix 1999; McGaugh et al. 2000; Verheijen 2001; Barton et al. 2001; Kannappan et al. 2002, and additional references therein). In a recent analysis, Kannappan et al. (2002) demonstrate that TF residuals correlate strongly with star formation indicators - color and $\mathrm{EW}(\mathrm{H} \alpha)$ — in the Nearby Field Galaxy Survey (NFGS, Jansen et al. 2000b; Kannappan et al. 2002), a statistically representative survey of all galaxy types with no bias against interacting, peculiar, or early-type galaxies. The inclusion of such galaxies distinguishes the NFGS TF sample (and most high- $z$ TF samples) from the majority of low- $z$ TF samples, which restrict analysis to a limited range of morphologies that may show only weak correlations between color and TF residuals (e.g., Courteau \& Rix 1999, see Kannappan et al. 2002 for further discussion). However, the Ursa Major cluster sample of Verheijen \& Sancisi (2001), which approximates a volume-limited sample, shows a stronger color-TF residual correlation (Verheijen 2001; Kannappan et al. 2002), and Bershady et al. (1999) also find initial evidence for a color-TF residual correlation at high $z$. The existence of this correlation implies that high- $z$ samples that differ in average color because of different selection criteria will also differ in average TF zero-point offset. If high- $z$ galaxies follow the same color-TF residual relation the NFGS follows, then we can use this relation to correct high- $z$ TF offsets for any bias toward blue colors (or we can use the $\mathrm{EW}(\mathrm{H} \alpha)-\mathrm{TF}$ residual relation to correct for any bias toward strong emission lines). Moreover, once such biases are removed, we can compare the remaining zero-point offset with the luminosity evolution predicted by the color-TF residual relation (based on true differences in mean color between high and low $z$ ), in order to determine whether TF zero-point evolution includes not only luminosity evolution, but also additional evolution reflecting the growth of stellar-tototal mass fractions over cosmic time (Kannappan et al. 2003a).

Obtaining a well-defined color-TF residual (CTFR) relation and measuring evolutionary offsets reliably may require special attention to galaxies with distorted or radially truncated rotation curves. In this paper, we demonstrate such an analysis at low $z$ using the Close Pairs Survey of Barton et al. (2001). The interacting galaxies in this survey display luminosity enhancements and misleading velocity offsets much like high- $z$ galaxies, as previously shown by Barton et al. (2001). However, at low $z$ we can use high-resolution kinematic data to identify problem rotation curves objectively, using quantitative tests of radial truncation and asymmetry of shape based on those introduced by Kannappan et al. (2002). Without explicitly accounting for kinematic anomalies, Barton et al. could not decouple luminosity offsets from velocity offsets and found no statistically significant CTFR relation for the Close Pairs Survey. We recover the CTFR relation for the Close Pairs Survey by eliminating galaxies with severely truncated or asymmetric rotation curves based on quantitative criteria, and by analyzing modestly asymmetric rotation curves with a robust velocity width measure that does not assume a functional form. Using these procedures, we find that the TF and CTFR relations for the Close Pairs Survey look very similar to the corresponding relations for the NFGS. Furthermore, the tightness of the Close Pairs Survey CTFR relation suggests that if a similar relation holds at higher $z$, determining whether galaxies lie on or off its locus may serve as a way to distinguish reliable luminosity evolution from TF offsets associated with kinematic anomalies.

Below, we describe the Close Pairs Survey and the NFGS $(\S 2)$, as well as our analysis methods ( $\$ 3)$, including quantitative criteria for identifying strongly asymmetric or truncated rotation curves. We then analyze the TF and CTFR relations for the Close Pairs Survey, with attention to kinematic anomalies, and compare the Close Pairs Survey relations to the corresponding NFGS relations ( $\$ 4)$. In $\S 5$ we examine the possible drivers of kinematic anomalies. We go on to consider the implications of our results for high- $z$ TF studies in $\S 6$. Finally, we summarize our conclusions in $\S 7$.

\section{DATA}

Our analysis makes use of two statistical surveys drawn from the CfA redshift surveys (Geller \& Huchra 1989): the Nearby Field Galaxy Survey (Jansen et al. 2000a,b; Kannappan et al. 2002), representing the general galaxy population, and the Close Pairs Survey (Barton et al. 2000a, 2001), representing galaxy pairs with line-of-sight velocity separation $\Delta V<1000$ $\mathrm{km} \mathrm{s}^{-1}$ and projected spatial separation $\Delta X \lesssim 100 \mathrm{kpc}$ (the distance limit differs from the original reference because we quote all distances and magnitudes using $\mathrm{H}_{0}=75 \mathrm{~km} \mathrm{~s}^{-1} \mathrm{Mpc}^{-1}$ and correct both surveys for Virgocentric infall following Jansen et al. 2000 and Kraan-Korteweg et al. 1984). These two surveys were selected without explicit bias in morphology or global environment. The Close Pairs Survey reflects the inherent luminosity bias of its magnitude-limited parent survey and also explicitly excludes galaxies with redshifts below $2300 \mathrm{~km} \mathrm{~s}^{-1}$. In 
contrast, the NFGS was selected with a greater representation of low-luminosity galaxies, in an effort to reproduce the local galaxy luminosity function (Jansen et al. 2000b); in practice, the NFGS luminosity distribution varies slowly over the range $-16>M_{B}>-22$ and cuts off for brighter and fainter galaxies, with emission-line galaxies naturally favoring lower luminosities within the sample (see Kannappan et al. 2002). Both surveys transmit the surface-brightness bias of their parent surveys, although the NFGS selection procedure was designed to minimize this bias (Jansen et al. 2000b).

For TF analysis we use one-dimensional optical emissionline rotation curves obtained with the FAST spectrograph on the 60-inch Tillinghast telescope at Mt. Hopkins (NFGS) and the Blue Channel spectrograph on the pre-conversion MMT (Close Pairs Survey and a few NFGS galaxies), as described in Barton et al. (2001) and Kannappan et al. (2002). Besides considering only emission-line galaxies, we further restrict our analysis to galaxies with $i>40$ and $\mathrm{M}_{\mathrm{B}}^{i}<-18$, except for a schematic look at TF outlier behavior among NFGS dwarfs in $\S 6$. We also require good alignment between the spectrograph slit and the galaxy major axis $(\triangle$ P.A. $<10$ for the NFGS and $\Delta$ P.A. $<20$ for the Close Pairs Survey ${ }^{3}$ ).

Fig. 1 compares property distributions for the two TF samples, in luminosity, morphology, color, redshift, surface brightness, and global environmental density. Sample properties are broadly similar, except for the luminosity and redshift distributions, which reflect the different selection methods discussed above. Notably, the choice of local pair environments does not strongly affect the distribution of global density environments for Close Pairs Survey galaxies, though these galaxies may show a slight underrepresentation of the lowest density environments compared to the NFGS (Fig. 1f). The requirement of detectable emission lines implies that within the NFGS and the Close Pairs Survey, the subsamples used for TF analysis have a higher proportion of low-density environments, as many cluster galaxies lack significant emission. Most high- $z$ TF samples also emphasize field environments, for similar reasons.

The NFGS and the Close Pairs Survey have three TF galaxies in common (A00442+3224, A22551+1931N, and NGC 7537). For these three galaxies, raw velocity widths from optical rotation curves are in excellent agreement, all within $\sim 8 \mathrm{~km} \mathrm{~s}^{-1}$. We also find reasonable agreement in rotation curve structure, despite different rotation curve extraction techniques (§ 3.2.3). Redshifts agree within $15-50 \mathrm{~km} \mathrm{~s}^{-1}$, and effective colors agree within 0.02 mag. Total B-band magnitudes agree within 0.15 mag. The only parameter for which the two surveys do not track closely is inclination angle (or equivalently, axial ratio), where we find differences of $10-20^{\circ}$ in both directions for the three common galaxies. Close Pairs Survey inclinations derive from careful analysis of new CCD data, whereas NFGS inclinations derive from low-precision diameter measurements tabulated in the UGC (Nilson 1973).

\section{METHODS}

\subsection{Tully-Fisher Analysis Techniques}

To facilitate direct comparison of the two surveys, we recompute the velocity widths and inclinations for the Close Pairs Survey (Barton et al. 2001, hereafter B01) with the methods used for the NFGS (see Kannappan et al. 2002, hereafter K02), and we correct the Close Pairs redshifts for Virgocentric infall to match the NFGS ( $\$ 2)$. We calculate extinction corrections for both surveys using the results of Tully et al. (1998) as described in K02, but without K02's special treatment of S0 galaxies.

The new inclinations, extinction corrections, and redshifts have quantitative, but no qualitative effects on the results. However, the new velocity widths do lead to some qualitative differences, because the "probable min-max" velocity measure $V_{p m m}$ adopted by K02 following Raychaudhury et al. (1997) is more robust than the velocity measure $V_{2.2}$ adopted by B01 following Courteau (1997). $V_{2.2}$ requires that a rotation curve conform to a standard functional form, which may provide a poor fit to rotation curves distorted by interaction. In contrast, $V_{p m m}$ uses all of the data points without imposing a particular model. As in K02, we define $V_{p m m}=0.5\left(V_{p \max }-V_{p \min }\right)$, where $V_{p \max } / p_{\text {min }}$ is defined as having a $10 \%$ chance of exceeding/lying below all velocities in the rotation curve. Each data point is modeled as a Gaussian distribution about the measured value, with $\sigma$ equal to the measurement error (see K02 for formulae). Using $V_{p m m}$, the pattern of TF offsets changes in such a way as to clarify the correlations reported in $\S 4.1$. (The parameter $W_{V_{p m m}}^{i}$ is related to $V_{p m m}$ via an inclination correction and a linear transformation that puts it on the same scale as the $W_{50}$ linewidths of radio observers, see K02.)

B01 and K02 have discussed the pros and cons of various TF fitting techniques. To avoid slope bias, we adopt an unweighted "inverse" fit (minimizing residuals in velocity) as our primary technique. A bias-corrected "forward" fit (Willick 1994) would give similar results, but such a fit would be very difficult to implement for the NFGS because of the intricacy of the survey's statistical selection procedure (Jansen et al. 2000b). In $\S 4.1$ we consider the effect of using a bias-corrected forward fit for the Close Pairs Survey, following the methods of B01, and we find no significant change in the results. Note that kinematically anomalous galaxies ( $\$ 3.2)$ are excluded from all TF fits, although they appear with special symbols in TF plots.

Ideally, we would like to compute the TF residuals used for the CTFR relation according to the procedure described in $\mathrm{K} 02$, in which the reference TF relation is defined by a fit over intermediate luminosities $\left(-17>\mathrm{M}_{\mathrm{B}}^{i}>-21\right)$ to avoid bias from high- and low-luminosity galaxies whose TF scatter is asymmetric. Unfortunately, this procedure is not practical for the Close Pairs Survey, both because the survey was not intended as a complete or representative sample of TF galaxies, and because the survey includes few galaxies fainter than $\sim-19.5$. In fact, the low-luminosity cutoff we apply to the NFGS at -18 is not necessary for the Close Pairs Survey, which includes no galaxies fainter than -18 because of its low-redshift cutoff $(\$ 2)$. We therefore analyze TF residuals for the Close Pairs Survey in two ways: (1) purely internally, i.e. determining both the TF relation and the CTFR relation from the Close Pairs Survey itself, with no luminosity cuts ( $\$ 4.1)$; and (2) using the NFGS as a

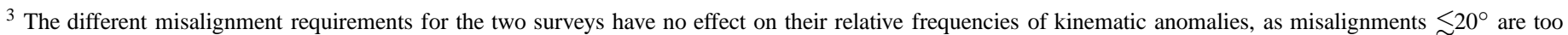

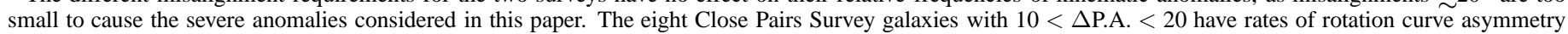
and rotation curve truncation consistent with overall rates for the survey as a whole.
} 
reference, i.e. determining the CTFR relation using Close Pairs TF residuals measured relative to the NFGS TF relation, again with no upper luminosity cuts (but with the NFGS limited to $\left.M_{B}^{i}<-18\right)$ to simplify comparison $(\S 4.2)$.

\subsection{Identifying Kinematically Anomalous Galaxies}

We refer to galaxies whose rotation curves are severely truncated in extent or highly asymmetric in shape as kinematically anomalous. Fig. 2 shows several examples of both anomalous and normal rotation curves. The next two sections discuss the criteria we use to flag kinematic anomalies. Both B01 and K02 have described methods for identifying anomalous rotation curves; here we adapt the methods of K02, who define continuous, quantitative measures of truncation and asymmetry. Although these measures are objective, the cutoff values of asymmetry and truncation used to reject galaxies must be empirically determined in order to optimize the rejection of TF and CTFR outliers for a given sample. Furthermore, strong asymmetries and severe truncation rarely coexist ( $\$ 3.2 .2$ and 5), so both types of anomaly must be considered in order to reject outliers successfully. We note that our empirical approach does not depend on whether the observed anomalies are intrinsic to the target galaxies or just artifacts of the data; however, we will argue in $\$ 5$ that at least some anomalies are intrinsic and discuss their physical origin.

\subsubsection{Rotation Curve Truncation}

To evaluate rotation curve truncation, we consider the average of the radial extents on the two sides of the rotation curve. (This average measure is slightly more robust than the one-sided measure used by K02.) For a pure theoretical exponential disk, the rotation curve will reach maximum velocity at 2.2 disk scale lengths or $\sim 1.3 r_{e}$ (Freeman 1970), so a rotation curve extent of less than $1.3 r_{e}$ could be considered suspect. In practice however, galaxies with rotation curves extending to $\sim 1.0-1.3 r_{e}$ are not outliers in our TF and CTFR relations, so we flag galaxies as anomalous only if their rotation curves are truncated at $<0.9 r_{e}$. Because measurements of $r_{e}$ are sensitive to details of profile extrapolation, the exact cutoff used to identify truncated rotation curves should be determined within a given TF data set.

\subsubsection{Rotation Curve Asymmetry}

Following K02, we define rotation curve asymmetry as the mean absolute deviation between velocities on the two sides of the rotation curve, expressed as a percentage of the velocity width $2 V_{p m m}$. This definition quantifies asymmetries in velocity structure between the two sides of the curve, e.g., due to one side rising and the other falling. We measure asymmetries by a procedure that involves numerically searching for the coordinate center of the rotation curve that minimizes the inner asymmetry (inside $1.3 r_{e}$ ) within certain constraints (for full details see K02). A key constraint is that the spatial center must stay within the error bars of the continuum peak position. ${ }^{4}$ Therefore galaxies in which the continuum center and the gas kinematic center do not agree tend to have large asymmetries. In essence, the asymmetry index combines a measure of shape asymmetry with a measure of the offset between the center of stellar light and the center of gas motion, due to dynamical disequilibrium or possibly extreme dust extinction. Note that the choice of center has no effect on $V_{p m m}$ or on our measure of rotation curve truncation, which averages the spatial extent on the two sides of the rotation curve.

We adopt a purely empirical definition of "strong" rotation curve asymmetry, based on the observed asymmetry distributions for both surveys (Fig. 3a). Most galaxies have asymmetries $\lesssim 8 \%$, but a few form a higher asymmetry tail to the distribution. These galaxies also scatter outside the rotation curve asymmetry-luminosity correlation reported by K02 (Fig. 3b). However, we find that most galaxies with moderately strong rotation curve asymmetries (8-10\%) and no rotation curve truncation follow the TF and CTFR relations, so we flag galaxies as anomalous only for rotation curve asymmetries $>10 \%$.

Notably, strong asymmetries are rare among the most truncated rotation curves, although there is no clear correlation between asymmetry and rotation curve extent (Fig. 4). The pattern in Fig. 4 is consistent with the view that severe truncation and strong asymmetries arise from related physical causes $(\S 5)$, but the most extreme truncation leads to a loss of information, where rotation curves may simply have inadequate radial extent to reflect asymmetries that would otherwise be significant.

Measuring rotation curve asymmetries reliably also requires adequate spatial resolution. Fig. 5 illustrates the effect of degrading resolution for the Close Pairs Survey. We show rotation curve asymmetries determined with the original spatial sampling of 0.6" per pixel (1-2" seeing) and with the data binned by 2 and by 4 . At the lowest resolution, some information is lost and occasionally the code crashes with insufficient data. We adopt the binned by 2 results for this paper because higher resolution is not available for two of the Close Pairs Survey galaxies, and because there are only minor differences between the binned by 2 and binned by 1 results.

For the NFGS, most rotation curves were binned on readout to 2.27 " per pixel (2" seeing). While this resolution is lower than the resolution used for the Close Pairs Survey, the loss of information is mostly offset by the fact that the NFGS TF sample is $\sim 1.7$ times closer in median redshift than the Close Pairs TF sample (Fig. 1d).

\subsubsection{Reproducibility of Truncation and Asymmetry Measures}

We evaluate the reproducibility of our truncation and asymmetry measures by comparing results for the three galaxies common to the NFGS and the Close Pairs Survey (observed with the FAST/60-inch combination for the NFGS and the Blue Channel/pre-conversion MMT combination for the Close Pairs Survey). Raw rotation curves from the two data sets agree well (Fig. 6). In two cases we see small deviations in the inner rise region that probably reflect differences in spatial resolution and rotation curve extraction technique between the surveys. ${ }^{5}$ Despite these small deviations, asymmetry measurements show the

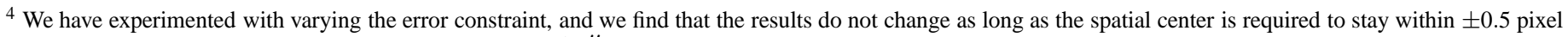

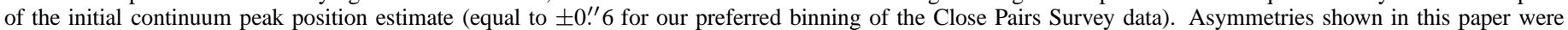

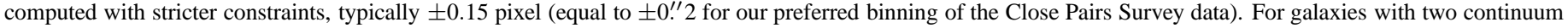

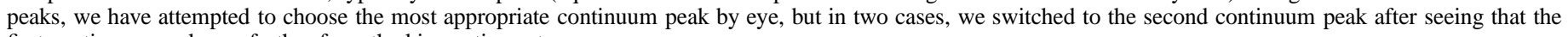
first continuum peak was further from the kinematic center.

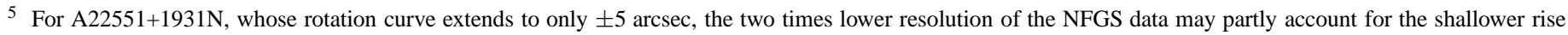

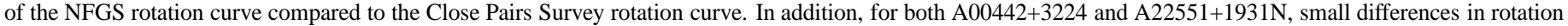

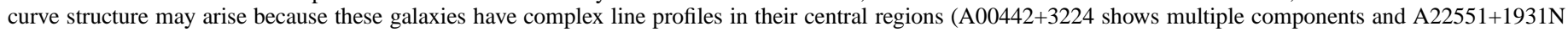

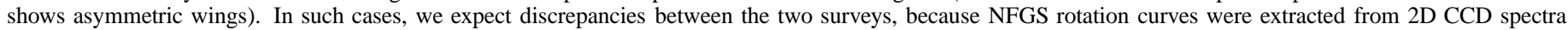


same general pattern in both data sets.

Truncation results also agree, in the sense that none of the rotation curves in either data set falls short of the cutoff radius at $0.9 r_{e}$ (shown in Fig. 6 with dashed and dotted gray lines for the Close Pairs Survey and NFGS respectively). However, we see up to $30 \%$ disagreement in measured $r_{e}$, presumably due to different techniques of photometric profile extrapolation. Rotation curve extents also differ, with NFGS rotation curves generally extending further. Examination of the raw data indicates lower $\mathrm{S} / \mathrm{N}$ in the Close Pairs data. The individual points in the NFGS rotation curves would have to have $\sim 4 \times$ lower S/N to yield similar rotation curve extents for the two surveys (with the NFGS rejection threshold set at $\mathrm{S} / \mathrm{N}=3$ and the Close Pairs rejection threshold set at cross-correlation $\mathrm{R}=2){ }^{6}$ These survey differences confirm that estimates of rotation curve truncation are subject to noise and systematic effects. We therefore reiterate that the exact rotation curve truncation threshold used for rejection should be determined within a given TF data set.

\section{TULLY-FISHER RESULTS}

This section discusses $\mathrm{TF}$ results obtained by excluding galaxies with severely truncated or asymmetric rotation curves. We defer interpretation of these kinematic anomalies to $\S 5$.

\subsection{The Close Pairs Survey}

Because the previous analysis of B01 revealed no significant correlation between color and TF residuals for the Close Pairs Survey, we must first demonstrate that our claim of a CTFR relation in the Close Pairs Survey is robust. Below we show how our treatment of kinematic anomalies allows us to detect the CTFR relation, in particular because of the very close correspondence between kinematically anomalous galaxies and CTFR outliers (which constitute $\sim 10 \%$ of the Close Pairs Survey TF sample).

Fig. 7 shows the TF and CTFR relations for the Close Pairs Survey, analyzing the survey purely on its own. The TF and CTFR fits exclude galaxies with highly truncated or asymmetric rotation curves (extent $<0.9 r_{e}$ or asymmetry $>10 \%, \S 3.2$ ), which are marked with triangles and circles respectively. Without these kinematically anomalous galaxies, the CTFR relation emerges clearly. Defining TF residuals relative to the inverse-fit TF relation shown by the solid line, a Spearman rank test gives $6.5 \times 10^{-11}$ probability of no correlation. Using a bias-corrected forward-fit TF relation based on the methods of B01 also yields a strong CTFR relation (no-correlation probability $1.3 \times 10^{-7}$ ), because the forward-fit and inverse-fit TF slopes are very similar (solid and dotted lines in Fig. 7, both shown with the zero point from the inverse fit. ${ }^{7}$ )

As discussed by B01 and K02, TF residual correlations like the CTFR relation should be tested rigorously, since any parameter that depends on luminosity will correlate with TF residuals if the TF slope is incorrect. K02 describe a fitting algorithm that avoids this problem, but this algorithm is not ideal for a sample like the Close Pairs Survey with a top-heavy luminosity distribution ( $\$ 3.1)$. Therefore we adopt the strategy of B01, using the robustness of the CTFR relation under changes of slope as a sanity check. The dashed line in Fig. 7 shows the slope required to eliminate the CTFR relation (i.e., to increase the probability of no correlation to $10 \%$ in a Spearman rank test). This slope is implausibly shallow and does not even pass through the bright end of the TF relation. Even the fit including kinematically anomalous galaxies (gray dot-dashed line) is significantly steeper, confirming the reality of the CTFR relation.

Compared to sigma-clipping applied to the TF relation (e.g., B01), our technique of identifying anomalous galaxies from their rotation curve properties is very effective at isolating galaxies whose TF residuals do not follow the CTFR relation. Most kinematically anomalous galaxies are CTFR outliers. However, TF outliers and CTFR outliers do not always correspond. In particular:

- Some kinematically anomalous galaxies are CTFR outliers, but not TF outliers. For example, the two CTFR outliers labeled $\mathrm{N}$ and $\mathrm{O}$ have very red colors and truncated rotation curves. Because these factors cause partially canceling TF offsets, these galaxies fall within the general cloud of TF scatter in spite of their kinematic abnormality. However, their TF residuals are actually incorrect for their colors, so they do not follow the CTFR relation. Likewise, galaxy $\mathrm{P}$ deviates slightly from the CTFR relation but remains within the cloud of TF scatter.

- The TF outliers labeled F and K in Fig. 7 are not CTFR outliers, nor are they flagged as kinematically anomalous. Their large TF residuals are actually in line with expectations based on their extremely blue colors, so they appear to define a young-starburst extension of the CTFR relation. Whether their TF residuals are actually reliable is unclear: indeed, galaxy $\mathrm{K}$ has a moderately high rotation curve asymmetry that would have reached $10 \%$ if we had adopted a higher-resolution asymmetry measure $(\S 3.2)$. These galaxies may have recently relaxed onto the CTFR relation from an earlier state of more severe kinematic disturbance.

In addition to our rejection strategy, our method of measuring velocity widths is also essential for defining a tight Close Pairs Survey CTFR relation. We use a robust velocity width measure, $V_{\text {pmm }}$, that yields reliable TF offsets even when rotation curves are somewhat asymmetric, below our $10 \%$ rejection threshold. K02 find that for disturbed or otherwise non-canonical rotation curves, $V_{p m m}$ produces more reliable results than $V_{2.2}$, the measure adopted by B01 following Courteau (1997). As a result, we find a more meaningful pattern of TF outliers than B01. Consider the eight TF outliers identified by B01 (labeled A-H in Fig. 7): (i) five are still TF outliers in our analysis, and they are both kinematically anomalous and CTFR outliers (C, D, E, G, and $\mathrm{H}$ ); (ii) two are no longer strong TF outliers in our analysis, and they are neither kinematically anomalous nor CTFR outliers (A and B; notably these two are the only galaxies for which B01 could not find a physical basis for outlier behavior); (iii) one is still a TF outlier, but its offset is consistent with

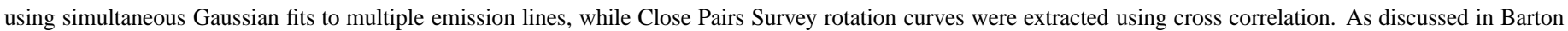

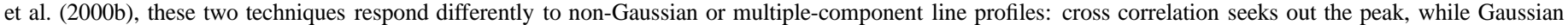
fitting finds something closer to an emission-weighted average.

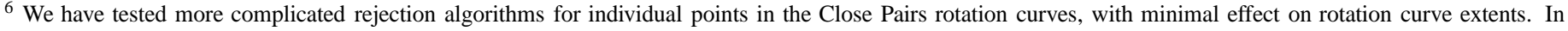
particular, truncated rotation curves remain truncated.

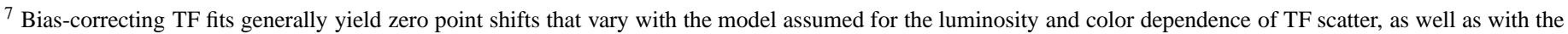
details of how measurement errors influence sample selection. This zero point shift has no relevance to our analysis, so we choose not to model it. 
a strong starburst that follows the CTFR relation, and it is not flagged as kinematically anomalous (F). Using $V_{p m m}$ also reveals several new examples that confirm the close correspondence between kinematic anomalies and CTFR outliers, independent of whether a galaxy lies on the TF relation $(\mathrm{I}, \mathrm{J}, \mathrm{K}, \mathrm{N}, \mathrm{O}, \mathrm{P}){ }^{8}$

\subsection{Comparison of the Close Pairs Survey and the NFGS}

We now turn to a comparative analysis of the Close Pairs Survey and the NFGS, to see whether the TF and CTFR relations for the Close Pairs Survey reveal evidence of interactioninduced luminosity enhancements relative to the general galaxy population (represented by the NFGS, Jansen et al. 2000b). Fig. 8 compares the inverse-fit TF relations for the NFGS and the Close Pairs Survey. The formal fit results yield a slope difference, with a slope of $7.93 \pm 0.29$ for the Close Pairs Survey (solid black line) and a steeper slope of $9.61 \pm 0.41$ for the NFGS (solid gray line), excluding kinematically anomalous galaxies in both cases. The slope difference is formally significant at $3.3 \sigma$ confidence. The size and significance of this difference agree with the results of B01 and may in part reflect enhanced star formation at the faint end of the TF relation.

However, in the region of Fig. 8 where the NFGS and the Close Pairs Survey overlap, the data look very similar (excluding kinematically anomalous galaxies). In fact, we find that the entire slope difference comes from two sources: (1) galaxies $\mathrm{F}$ and $\mathrm{K}$, and (2) the difference between the two surveys' luminosity distributions (Fig. 1 and $\S 2$ ). We argue below that galaxies $\mathrm{F}$ and $\mathrm{K}$ may be the only galaxies in the Close Pairs Survey with significant interaction-driven luminosity boosts that are not accompanied by severe kinematic anomalies. Without galaxies $\mathrm{F}$ and $\mathrm{K}$, the Close Pairs Survey TF relation would have a slope of 8.8 (dotted line). Likewise, if we weight each data point in the NFGS TF relation by the ratio of the two surveys' luminosity distributions (i.e., the ratio of the histograms shown in Fig. 1, but with kinematically anomalous galaxies removed), the resulting TF fit yields a slope of 8.8. We conclude that the two surveys' TF relations are quite similar, except for a population of disturbed galaxies within the Close Pairs Survey, which includes galaxies $\mathrm{F}$ and $\mathrm{K}$ as well as the galaxies we have rejected because of severe kinematic anomalies (not all of which are TF outliers). We stress that this disturbed population almost certainly reflects enhanced star formation from interactions, consistent with B01. However, quantitatively separating luminosity and velocity offsets is impossible for most of these galaxies.

Nonetheless, we do see possible evidence for pure luminosity boosts in the Close Pairs Survey, based on the position of galaxies $\mathrm{F}$ and $\mathrm{K}$ in the CTFR relation. To construct the CTFR relations for the Close Pairs Survey and the NFGS in Fig. 8, we compute the TF residuals for both surveys relative to the NFGS TF relation, which represents the TF relation for the general galaxy population. Comparing the two CTFR relations, we find very similar locii, except for an extension of the Close Pairs Survey CTFR relation toward bluer colors. Blueward of $(B-R)_{e}=0.6$, there are four Close Pairs Survey galaxies, but no NFGS galaxies. If the frequency of very blue galaxies in the Close Pairs Survey were representative of the general galaxy population, the probability of the NFGS containing zero very blue galaxies would be $3 \%$ in a random sample, implying that the difference between the surveys is probably real. Two of the very blue Close Pairs Survey galaxies are kinematically anomalous, but the other two (galaxies F and K) lie on the CTFR relation, extending it as far as $(B-R)_{e} \sim 0.3$.

These two galaxies' $\sim 2.5$ mag TF residuals probably reflect luminosity enhancements from starbursts. We cannot rule out kinematic effects, especially for galaxy $\mathrm{K}(\S 4.1)$, but the fact that both galaxies have objectively acceptable kinematic anomalies and also fall on the CTFR relation is reassuring. As argued by $\mathrm{K} 02$, the slope of the CTFR relation in optical passbands is consistent with the slope expected when the dominant physics determining TF offsets involves star formation. Population synthesis models combined with a variety of galaxy formation models all yield similar predictions for the slope of the relation between color and stellar mass-to-light ratio (Bell \& de Jong 2001), which may be converted to predictions for the slope of the CTFR relation given certain simplifying assumptions (e.g., that dark matter fractions are constant). The predicted and observed slopes agree well, suggesting that star formation explains most of the CTFR slope (K02). In this context, we interpret the blue extension of the Close Pairs CTFR relation as evidence of very bright, young starbursts driven by interactions. For reasonable star formation histories, even $10 \%$ mass starbursts generally extend rather than depart from the CTFR relation (Bell \& de Jong 2001, see their Fig. 5). After 1 Gyr, such bursts tend to fall slightly above the CTFR relation, but still within the observational scatter set by our measurement errors.

Younger bursts may show more extreme luminosity offsets that do not follow the CTFR relation. Unfortunately, most CTFR outliers have kinematic anomalies, so we cannot readily disentangle luminosity and velocity offsets for these galaxies. Even for those few CTFR outliers without kinematic anomalies, velocity offsets may play a role. All but one of the nonanomalous NFGS CTFR outliers labeled $w-z$ in Fig. 8 have companions that could be causing luminosity offsets, but these same companions may also cause velocity offsets, for example via systematic inclination errors from photometric distortion. In one case we also suspect a large asymmetry hidden by a poorly sampled rotation curve.

K02 find that integrated $\mathrm{H} \alpha$ equivalent widths (i.e., with the spectrograph slit scanned over the entire galaxy Jansen et al. 2000a) also correlate with TF residuals for the NFGS, and Fig. 9 shows that galaxies $w, y$, and $z$ are outliers from the integrated $\mathrm{EW}(\mathrm{H} \alpha)-\mathrm{TF}$ residual relation just as they are from the color-TF residual relation. However, the integrated $\mathrm{EW}(\mathrm{H} \alpha)-\mathrm{TF}$ residual relation is more scattered than the CTFR relation, and the status of its outliers is less obvious. Moreover, the use of central rather than integrated $\mathrm{EW}(\mathrm{H} \alpha)$ measurements degrades the relation considerably (compare both panels of Fig. 9). Unfortunately, central measurements are the only type available for the Close Pairs Survey. Although some CTFR outliers remain outliers in the central $\mathrm{EW}(\mathrm{H} \alpha)-\mathrm{TF}$ residual relation, the latter relation does not offer a clean way to separate offsets affected by kinematic anomalies from reliable luminosity boosts.

Most blue starburst galaxies in the Close Pairs Survey are fainter than $\mathrm{M}_{\mathrm{B}} \sim-21$, despite the overall survey bias toward bright galaxies (e.g., Fig. 7). The NFGS shows a similar tendency, resulting in asymmetric scatter at the bright end of the $\mathrm{TF}$ relation (K02). The absence of very blue galaxies at high lu-

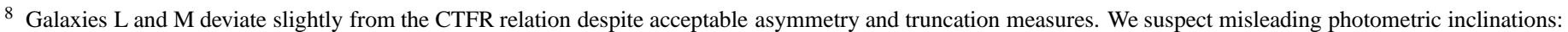

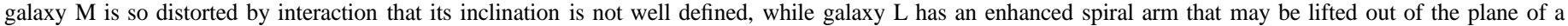


separate kinematic system on one side (Fig 2).
} 
minosities may reflect a hierarchical formation history in which bright galaxies form early via mergers that consume most of the available gas, so that in later interactions these galaxies lack the fuel necessary for major starbursts. B01 show that the Close Pairs data are consistent with interaction-driven starbursts of relatively constant size, so that the fractional contribution of the starburst light to the total light is negligible for the largest galaxies.

\section{THE ORIGINS OF KINEMATIC ANOMALIES}

Kinematically anomalous galaxies with severely truncated and/or asymmetric rotation curves represent nearly $20 \%$ of the Close Pairs Survey TF sample, roughly twice the frequency seen for the corresponding NFGS sample. The bulk of the difference arises from the 3-4× higher incidence of strong rotation curve asymmetries in the Close Pairs Survey (10/88 vs. 2/73). Truncated rotation curves also occur more often in the Close Pairs Survey (9/88 vs. $5 / 73)$, but with only marginal statistical significance. The significance of the frequency difference for strong rotation curve asymmetries depends upon interpretation. A K-S test finds no significant difference between the two continuous asymmetry distributions shown in Fig. 3. However, accepting that asymmetries above 8-10\% are "anomalous," i.e., represent a discontinuously disturbed population, the difference in the rate of anomalies between the two surveys is highly significant. For example, if we assume that the NFGS reflects the underlying parent distribution of asymmetries, the probability of obtaining rotation curve asymmetries $>10 \%$ in 10/88 Close Pairs Survey galaxies is $3.6 \times 10^{-7}$. In reality, systematics dominate this problem, and statistical tests are of limited utility. Confirmation with other samples would be more valuable.

Most of our kinematically anomalous galaxies have either strongly asymmetric or truncated rotation curves, but not both. However, rotation curve truncation may prevent detection of non-central asymmetries (§3.2.2). Fig. 8 shows that large rotation curve asymmetries generally occur in blue galaxies, while truncated rotation curves generally occur in red galaxies (though the latter may have relatively blue centers, $\S 5.2$ ).

\subsection{Sources of Rotation Curve Asymmetries}

The fact that strong rotation curve asymmetries occur more often in the Close Pairs Survey than in the NFGS provides circumstantial evidence that close neighbors play a role in driving such asymmetries. In their selection criteria, the two surveys differ primarily in local environmental distribution (pairs vs. any environment) and luminosity distribution (top-heavy vs. representing a broad range of luminosities, Fig. 1). If anything, the difference in luminosity distributions causes us to underestimate the difference in rotation curve asymmetries: the topheavy luminosity distribution of the Close Pairs Survey should not favor large rotation curve asymmetries, because luminosity and rotation curve asymmetry anticorrelate in the general galaxy population (K02 and NFGS symbols in Fig. 3b). We conclude that the higher rate of strong rotation curve asymmetries in the Close Pairs Survey very likely reflects the selection of close pair environments, barring any systematic difference in the data (unlikely given our conclusions regarding resolution dependence and reproducibility in $\S 3.2 .2-3.2 .3$ ).
Interactions with neighbors or generally dense environments may induce rotation curve asymmetries via several mechanisms. All rotation curves show some degree of asymmetry, which correlates with inclination, Hubble type, and galaxy color $^{9}$ (Fig. 10; Kannappan \& Fabricant 2001; Kannappan 2001; see also Beauvais \& Bothun 1999) and may be related to small satellite interactions or turbulence from self-regulated star formation. Strong asymmetries may arise from tidally disrupted gas and/or decoupling between the gas dynamical center of the galaxy and the stellar continuum peak position during violent encounters (e.g., Mihos 2001; Barton et al. 2001; Kornreich et al. 2002). Asymmetries may also reflect the presence of multiple gas components due to merging systems or strong bars, since standard rotation curve analysis techniques assign a single velocity per spatial position even when there are multiple velocity components, and which velocity component dominates the result may vary strongly with local changes in emission-line strength (Barton et al. 2000b). Rubin et al. (1999) find evidence that cluster interactions may produce long-lived disturbances in rotation curves (but see Dale et al. 2001); we note that our surveys contain few galaxies in dense cluster environments, because field environments are statistically more common (Fig. 1). Extinction in dusty starbursts can also cause rotation curve asymmetries, although in our surveys, most galaxies with large rotation curve asymmetries show blue colors atypical of dusty systems (Fig. 8).

We plan to investigate how these mechanisms combine to explain observed rotation curve asymmetries in a future paper using integral-field kinematics (with M. Bershady, in preparation). In the absence of understanding the origin of rotation curve asymmetries, we cannot at present explain their relationship to TF offsets. The large TF offsets we observe for galaxies with high rotation curve asymmetries may reflect concomitant luminosity enhancements and/or symmetric rotation curve distortions (Barton et al. 1999), in addition to the direct effects of rotation curve asymmetries.

\subsection{Sources of Rotation Curve Truncation}

The slight difference in rotation curve truncation frequency between the NFGS and the Close Pairs Survey may reflect nothing more profound than $\mathrm{S} / \mathrm{N}$. If we edit each NFGS rotation curve to remove individual points that would be lost at the $\mathrm{S} / \mathrm{N}$ of the Close Pairs Survey (using the relative S/N determined by comparing galaxies common to the two surveys in $\S 3.2 .3$ ), three additional NFGS rotation curves become truncated, so that the frequency of truncation is indistinguishable between the surveys. ${ }^{10}$

However, physical arguments predict an enhanced rotation curve truncation rate for galaxies selected in pairs (as discussed by B01). Neighbor interactions can trigger disk gas inflow and central starburst activity (e.g., Hernquist \& Mihos 1995; Barton Gillespie et al. 2003), causing optical emission lines to be preferentially detected at small radii. In addition, galaxies in cluster environments may show radially truncated emission due to gas stripping or tidal harassment (Gunn \& Gott 1972; Moore et al. 1998), though only a few galaxies in the Close Pairs Survey have sufficiently dense environments for cluster mechanisms to

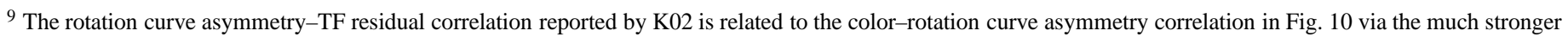
color-TF residual correlation.

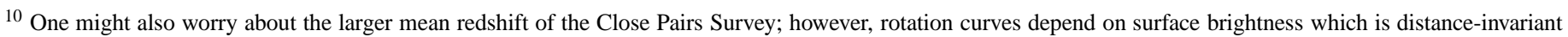

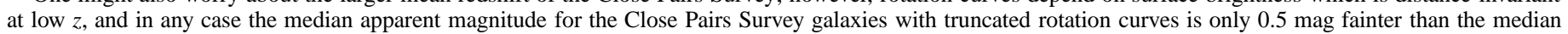
apparent magnitude for the NFGS. We see no evidence for systematically poor weather conditions during observations of galaxies with truncated rotation curves. 
play a role (and in particular, the galaxies with truncated rotation curves have modest densities on the scale of Fig. 1f, ranging from -0.2 to 0.5 on the $\log$ scale, with median -0.06 ).

These physical processes might have produced more truncated rotation curves in the Close Pairs Survey if the survey were not deficient in low-luminosity galaxies. Even with this deficiency, truncated rotation curves appear more common at lower luminosities within the survey (Fig. 7; the luminosity distributions for truncated and non-truncated rotation curves differ at $95 \%$ confidence in a K-S test). Moreover, physically induced rotation curve truncation may also be more common at lower luminosities, based on a two-step chain of inference. (1) Several Close Pairs Survey galaxies with truncated rotation curves have unusual color profiles suggestive of gas inflow processes: their colors within $r_{e}$ are bluer than their outer disk colors. In general, blue-centered galaxies include blue compact morphologies, such as blue-centered emission-line S0 galaxies, as well as many later-type morphologies, and they often show evidence of interactions and mergers (Kannappan et al. 2003b). Statistically, truncated rotation curves in the Close Pairs Survey correlate with blue-centered galaxies at $98 \%$ confidence (four of nine galaxies). (2) Blue-centered galaxies are nearly always fainter than $M_{B}=-20$ (Kannappan et al. 2003b). Thus rotation curve truncation associated with blue-centered galaxies will occur primarily at low luminosities. Because low-luminosity galaxies are underrepresented in the Close Pairs Survey compared to the NFGS $(\S 2)$, this trend probably weakens any statistical difference between the two surveys' rates of rotation curve truncation, insofar as that difference is related to interaction-driven gas inflow. Therefore in our view interactions remain a likely source of rotation curve truncation.

\section{IMPLICATIONS FOR HIGH- $z$ TF STUDIES}

The pattern of TF offsets for kinematically anomalous galaxies in the Close Pairs Survey looks very similar to the "differential luminosity evolution" seen in some high- $z$ TF studies (e.g., Simard \& Pritchet 1998; Ziegler et al. 2002). Fig. 11 shows the Close Pairs Survey and the NFGS alongside high- $z$ TF data from the FORS Deep Field (the survey used by Ziegler et al. 2002), courtesy of A. Böhm (Böhm et al. 2003). Böhm et al. (2003) derive velocities by fitting simulated RCs, including slit width and seeing effects, to observed (extracted) onedimensional RCs. For both the high- $z$ sample and the Close Pairs Survey, the largest TF offsets occur in the same region of parameter space, i.e., at luminosities fainter than $\mathrm{M}_{\mathrm{B}} \sim-21$ and velocity widths less than $\log 2 V \sim 2.2$. In both cases, this pattern of TF offsets creates a shallow TF slope compared to the reference slope defined by the NFGS.

Furthermore, at both high and low $z$, this pattern of offsets is linked to kinematic anomalies. In the Close Pairs Survey, the largest TF offsets correspond to kinematically anomalous galaxies, which have offsets of up to 4 mag. In the FORS Deep Field sample, much of the slope evolution is driven by "lowquality" data points, identified as such by Böhm et al. because the corresponding rotation curves "have a smaller radial extent and partly feature signatures of moderate kinematic perturbations like waves or asymmetries" (Böhm et al. 2003, data table notes; no quantitative criteria given). By analogy with the Close Pairs Survey, we suggest that the slope evolution in the FORS Deep Field may reflect kinematic anomalies caused by compan- ions or minor mergers. The frequency of interactions and mergers is expected to increase with $z$ (Patton et al. 2002; Murali et al. 2002), and physically significant interactions are not always visually obvious. In fact, many kinematically anomalous galaxies in the NFGS show only subtle interaction evidence, with faint or already merging small companions. Conversely, large, obvious galaxy pairs need not be influenced by interactions. ${ }^{11}$ The brightest galaxies in the Close Pairs Survey all have companions (by definition), yet they display minimal starburst activity and few strong kinematic anomalies, possibly because the galaxy formation process consumes most of the gas in massive galaxies early, inhibiting gas-dynamical processes in later interactions ( $\$ 4.2)$.

Interaction-induced kinematic anomalies may be more common at high $z$ than is generally recognized. Rotation curve asymmetries like those in Fig. 2 will not always be obvious at the resolution of high- $z$ data. Moreover, the high frequency of blue compact galaxies in many high- $z$ TF studies probably reflects interaction-driven starburst activity that can cause rotation curve truncation via gas inflow processes (B01 and Barton \& van Zee 2001). Low $\mathrm{S} / \mathrm{N}$ is another potential source of rotation curve truncation and thereby TF outliers in some high- $z$ studies, especially studies already biased toward centrally concentrated star formation (as Kannappan 2001, suggests for the Simard \& Pritchet 1998 study; see also Kobulnicky \& Gebhardt 2000).

To the extent that kinematically anomalous galaxies drive the apparent luminosity evolution in high- $z$ TF studies, the nature of that evolution is unclear. At present, disentangling luminosity and velocity offsets for galaxies with rotation curve asymmetries is not possible. Estimating velocity offsets for galaxies with rotation curve truncation is also difficult, although we do know that for blue compact galaxies in particular, underestimated velocity widths may yield average TF offsets of order 2 mag for unresolved optical data (based on studies of their low- $z$ analogues, Barton \& van Zee 2001; Pisano et al. 2001).

The exact effects of rotation curve truncation may differ at low and high $z$, because most high- $z$ studies do not compute velocity widths directly from rotation curves but instead analyze kinematic and photometric profiles together (e.g., Vogt et al. 1996; Simard \& Pritchet 1999; Ziegler et al. 2002). These modeling techniques generally assume that emission-line flux and disk-continuum flux profiles are simply related by scalings in radial extent and intensity. The models further assume a basic form for the rotation curve constrained by exponential fits to the spatial flux distribution, even when HST data are available to model bulge components in the images. Within these assumptions, using high- $z$ techniques may mitigate truncationinduced velocity offsets in the TF relation (although probably not asymmetry-induced velocity offsets). However, the presence of bulges, bars, central starbursts, and morphological distortions will severely compromise such modeling. A realistic evaluation of the effects of rotation curve truncation on high- $z$ data will require simulating high- $z$ resolution, $\mathrm{S} / \mathrm{N}$, and analysis techniques using a low- $z$ sample like the NFGS that includes all Hubble types, as well as barred, peculiar, and interacting galaxies.

In short, the contribution of velocity offsets to high- $z$ luminosity evolution is uncertain but potentially large. Discrepancies between high- $z$ TF studies that report minimal evolution

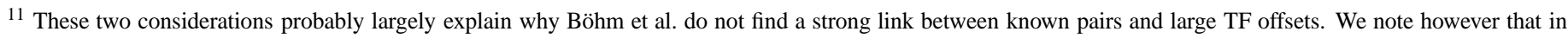

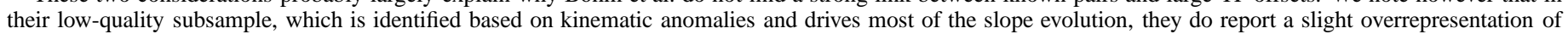
pair/cluster candidates among galaxies with large TF offsets. 
(e.g., Vogt et al. 1997; Bershady et al. 1999), and 1-2 mag evolution (e.g., Rix et al. 1997; Simard \& Pritchet 1998; Ziegler et al. 2002) may in part reflect differences in sample selection criteria that lead to higher or lower percentages of kinematically anomalous galaxies. For example, the Vogt et al. (1997) sample favors large, undisturbed disks, while the Ziegler et al. (2002) sample includes all types of elongated emission-line galaxies, and thus almost certainly galaxies with kinematic anomalies.

Measuring luminosity evolution reliably will require identifying and rejecting kinematically anomalous galaxies in high- $z$ samples and/or accounting for the velocity offsets inherent in these galaxies' TF offsets. Isolating TF evolution associated with kinematic anomalies may also be interesting in its own right, as a way to probe the evolving role of mergers and interactions as a function of luminosity and redshift. At low $z$, the NFGS shows a distinct population of kinematically anomalous galaxies fainter than $\mathrm{M}_{\mathrm{B}}=-18$ (Fig. 11), perhaps reflecting late-epoch galaxy formation activity on the smallest mass scales. Below we consider four possible strategies for analyzing high- $z$ TF data in the presence of kinematic anomalies.

\subsection{Identification Based on Rotation Curve Properties}

By definition, the most accurate way to identify kinematically anomalous galaxies is via their rotation curve properties. Spatially resolved rotation curves combined with good $r_{e}$ measurements should be sufficient to flag cases of rotation curve truncation, using a rejection threshold optimized for the TF sample under study ( $\$ 3.2 .1$ ). However, high- $z$ kinematic data generally lack the spatial resolution necessary to measure reliable rotation curve asymmetries (\$ 3.2.2). A new generation of 20-30-meter telescopes with adaptive optics may enable such measurements in the future.

\subsection{Identification Based on Morphology}

Most low- $z$ TF studies would reject the strongest TF outliers in the Close Pairs Survey on morphological grounds. Of the nine strongest TF outliers in the survey (TF residuals brighter than -1.5 mag in Fig. 7), three are emission-line S0 galaxies, while the other six are distorted by interactions, including the two that follow the CTFR relation. High- $z$ TF studies typically compare against low- $z$ calibration samples that exclude such early-type or disturbed morphologies. However, the high- $z$ samples themselves may not exclude such morphologies, due to sample selection procedures that rely on spectroscopic galaxy types and/or low physical-resolution images. For example, the abundance of compact narrow emission line galaxies (CNELGs, Guzman et al. 1996) in high- $z$ samples may in part reflect the inclusion of emission-line S0 galaxies. Barton \& van Zee (2001) show that four TF outliers in the Close Pairs Survey have properties that suggest they are counterparts to CNELGs at higher redshift; of these four, two are emission-line S0's and one is an early-type peculiar galaxy (according to the classifications of Kannappan et al. 2003b). Emission-line S0 galaxies form a prominent subpopulation of kinematically anomalous galaxies in the NFGS as well, as indicated by the asterisks in Fig. 11.

In general, all high- $z$ TF studies that report differential luminosity evolution employ selection criteria that allow emissionline S0 and disturbed spiral morphologies. With HST imaging, a strategy of rejecting both classes of galaxy might successfully eliminate many kinematic anomalies at high $z$ and establish whether the non-anomalous galaxy population shows differential luminosity evolution. However, some anomalous galaxies would probably escape rejection, while some nonanomalous galaxies would probably be thrown out, possibly including galaxies essential to measuring luminosity evolution like the two galaxies that seem to extend the CTFR relation for the Close Pairs Survey. Also, this approach would be too imprecise to support a detailed analysis of kinematically anomalous galaxies for their own sake.

\subsection{Identification Based on the Color-TF Residual Relation}

If a color-TF residual relation can be established at high $z$, then identifying kinematically anomalous galaxies based on their close correspondence with CTFR outliers may be easier than identifying them from their rotation curve properties, with comparable effectiveness for separating reliable luminosity evolution from the ambiguous TF offsets of kinematically anomalous galaxies. In the Close Pairs Survey, reliable luminosity offsets lie along the CTFR relation, possibly extending it toward bluer colors, while offsets that do not follow the CTFR relation nearly always correspond to kinematic anomalies.

With a large enough sample, it may be possible to establish a high- $z$ CTFR relation just using sigma clipping and rejection based on morphology or rotation curve truncation. Bershady et al. (1999) report initial evidence for a high- $z$ CTFR relation based on a sample of disk galaxies spanning a broad range of colors. The low- $z$ CTFR relation may be used to help refine the locus of the high- $z$ CTFR relation, assuming closely standardized analysis techniques, though we caution that the CTFR relation may evolve. If defining a tight high- $z$ relation proves difficult, establishing its locus might require a small sample of galaxies with well-resolved rotation curves, free of kinematic anomalies according to the criteria presented here. Obtaining such data at high $z$ would probably require deep ground-based spectroscopy with adaptive optics. Fortunately, once a high- $z$ CTFR relation has been established, kinematic anomalies can be eliminated from a larger sample with unresolved or poorly resolved rotation curves simply by rejecting CTFR outliers. Rejecting CTFR outliers can also help to eliminate galaxies with faulty inclination estimates or otherwise spurious data. Any reliable luminosity evolution that remains can then be measured by comparing the high- and low- $z$ CTFR relations, perhaps with the precaution of obtaining well-resolved rotation curves for any high- $z$ galaxies that extend the CTFR relation.

Since measuring equivalent widths is often easier than measuring colors at high $z$, attempting to identify kinematic anomalies using the $\mathrm{EW}(\mathrm{H} \alpha)$ - or $\mathrm{EW}([\mathrm{OII}])-\mathrm{TF}$ residual relations might also be worthwhile. These relations have already proven useful for estimating bulk luminosity shifts related to differences in mean emission-line strength between high- and low- $z$ TF samples (Kannappan 2001; Kannappan et al. 2003a, 2004). However, the emission-line strength-TF residual relations are noisier than the CTFR relation even at low $z$ (K02), and equivalent widths can depend strongly on spectroscopic aperture (§ 4.2, Jansen et al. 2000a). Thus it is not yet clear that emission-line strength can serve as a surrogate for color for identifying kinematically anomalous galaxies.

\subsection{Construction of Matching Low-z Calibration Samples}

An alternative to rejection at high redshift is greater inclusion at low redshift. For example, the NFGS includes kinematically anomalous galaxies similar to those at high $z$, because it is a statistically representative, morphology-blind sample of the local galaxy population. Many of the worst TF outliers in the NFGS 
are emission-line S0 or irregular late-type galaxies with kinematic oddities such as large rotation curve asymmetries, truncated rotation curves, or counterrotating gas and stars (K02; see also Kannappan \& Fabricant 2001). Including such kinematically anomalous galaxies in determining the low- $z$ reference TF relation could help to eliminate apparent evolution between low and high redshift that really reflects different sample selection criteria.

To first order, an all-inclusive low- $z$ reference sample should provide a good calibration for a magnitude-limited high- $z$ sample like the FORS Deep Field (Böhm et al. 2003). To illustrate, we compare TF fits for the NFGS, the FORS Deep Field, and the Close Pairs Survey in Fig. 11. The NFGS defines the reference relation, shown as a solid line in all three panels. The dashed lines show the best-fit TF relations for the other two samples with the slope held fixed to the NFGS value, where we include two lines for the FORS Deep Field sample to show fits with and without the data designated as "low-quality" by Böhm et al. (2003) because of kinematic perturbations or inadequate rotation curve extent. While we cannot directly assess whether Böhm et al.'s low-quality data points would meet our quantitative criteria for kinematic anomalies, the 0.18 mag difference between fits with and without these low-quality points gives some idea of the likely contribution of kinematically anomalous galaxies to the TF offset for the FORS Deep Field. This contribution is quite similar to the 0.16 mag offset for the Close Pairs Survey relative to the NFGS. Apart from the $0.18 \mathrm{mag}$ attributed to low-quality points, we find an offset of only 0.28 mag for the FORS Deep Field relative to the NFGS. Böhm et al. (2003) find a larger mean offset in part because they follow other high- $z$ workers in using the TF relation of Pierce \& Tully (1992) as a low- $z$ reference relation. This relation has a known zero-point error of $\sim 0.3-0.4 \mathrm{mag}$ (Tully \& Pierce 2000, page 776), depending on whether one prefers $\mathrm{H}_{0}=75$ or $70 \mathrm{~km} \mathrm{~s}^{-1} \mathrm{Mpc}^{-1}$. A high- $z$ TF offset of $\sim 0.4$ mag would be expected for the FORS Deep Field based on the sample's bluer mean color compared to the NFGS, assuming the same CTFR relation observed at low $z$ (A. Böhm, private communication). Thus the observed $\sim 0.3$ mag offset is consistent with a combination of evolution in mass-to-light ratio (as suggested by Böhm et al.) and perhaps some evolution in stellar-to-total mass fraction (as discussed for the Vogt et al. 1997 sample in Kannappan et al. 2003a). However, uncertainties and systematics dominate at this level: (i) scatter in the high- $z$ data, (ii) differences between velocity measures (e.g., using a $V_{\text {pmm }}$ equivalent at high $z$ could decrease the evolution by $\sim 0.1 \mathrm{mag}$ from what we measure, Kannappan 2001), (iii) differences in Galactic extinction corrections ${ }^{12}$, (iv) the unknown true frequency of kinematic anomalies at high $z$, and (v) differences in color or surface-brightness selection biases, which may affect the relative frequency of blue colors and/or kinematic anomalies (e.g., by favoring high surface brightness blue compact galaxies).

In addition to affecting mean offsets, kinematic anomalies also affect slope evolution. As previously discussed, the FORS Deep Field TF relation is shallower than the NFGS TF relation (inverse-fit slopes of -6.3 and -7.4 , respectively; the high- $z$ fit is shown as a dotted line in Fig. 11). This result confirms the slope evolution reported by Ziegler et al. (2002), although the exact slope differs because of different extinction corrections and fitting methods. Unlike Böhm et al., however, we do not find statistically significant slope evolution for the high-quality points taken alone. Separate inverse fits to the high and low quality data in Fig. 11 suggest that the shallower slope is produced almost entirely by the low-quality subsample, which is subject to kinematic anomalies. Specifically, the slope evolution is driven by anomalous galaxies with large TF offsets of up to $\sim 2$ mag at intermediate luminosities, $\mathrm{M}_{\mathrm{B}} \sim-18$ to -21 (Fig. 11). By comparison, NFGS galaxies with strong kinematic anomalies and large TF offsets typically have luminosities fainter than $M_{B}=-18$. Mallén-Ornelas et al. (1999) report an analogous evolutionary shift in the characteristic luminosities of blue compact galaxies from high to low $z$. These trends may reflect mass-dependent evolution in the rate of starbursts and gas-dynamical disturbances driven by galaxy mergers and interactions, following the hierarchical tendency for today's more massive galaxies to show peak formation activity at higher redshifts than today's smaller galaxies (e.g., Cowie et al. 1996). In this view, both the number density and the luminosity distribution of kinematically anomalous galaxies would be expected to evolve, and the optimal low-z calibration sample for isolating luminosity offsets from velocity offsets would be a sample that simulated the expected luminosity distribution of kinematic anomalies at higher $z$. The simulation would ideally involve adjusting both the interacting galaxy distribution and the data quality (e.g., rotation curve $\mathrm{S} / \mathrm{N}$, inclination errors) to match the high- $z$ sample under study. Modeling high $-z$ analysis techniques would also be essential, and such modeling might lead to development of a high- $z$ analogue of $V_{p m m}$ that would minimize velocity offsets from minor kinematic distortions.

\section{CONCLUSIONS}

We have demonstrated robust methods for measuring luminosity evolution in TF samples with a high frequency of rotation curve anomalies, such as might be expected at high redshift. The Close Pairs Survey of Barton et al. (2001) is ideal for this analysis, as a low- $z$ TF sample with high-quality data and many similarities to high- $z$ TF samples: optical emission-line rotation curves, morphology-blind selection, and a large number of interacting galaxies. The Nearby Field Galaxy Survey (NFGS, Jansen et al. 2000b; Kannappan et al. 2002) offers a low-z reference sample with similar features, but with a more typical number of interacting galaxies. We have extended Barton et al.'s previous TF analysis of the Close Pairs Survey, which showed that both starbursts and kinematic disturbances can create apparent "luminosity evolution" for galaxies in interacting pairs, by our demonstration of methods for isolating potentially spurious luminosity offsets associated with severe kinematic anomalies from reliable luminosity offsets clearly linked to star formation.

The largest apparent luminosity offsets in the Close Pairs Survey TF relation correspond to galaxies with severe kinematic anomalies (asymmetric rotation curve shapes and/or radially truncated rotation curve extents, using objective measures adapted from Kannappan et al. 2002). The pattern of these galaxies' TF offsets looks much like the differential luminosity evolution claimed in many high- $z$ studies, with the largest $\mathrm{TF}$ offsets at luminosities fainter than $\mathrm{M}_{\mathrm{B}} \sim-21$. Excluding the galaxies with asymmetric or truncated rotation curves, however, and adopting a robust velocity width measure insensitive to minor kinematic distortions, we find that the TF relations for the

12 We standardize internal but not Galactic extinction corrections (the FORS Deep Field corrections are described in Heidt et al. 2003). Applying our Galactic extinction conventions to the high- $z$ data would increase the evolution by $\sim 0.04-0.08$ mag from what we measure, depending on the passband closest to rest-frame B for a given galaxy (based on known zero point differences between different extinction maps, Burstein 2003). 
Close Pairs Survey and the NFGS are very similar, with no significant evidence for overall luminosity enhancement in paired galaxies relative to the general population.

Nonetheless, we do find evidence for luminosity enhancement when we compare the color-TF residual (CTFR) relations for the two surveys. Two galaxies that are not objectively flagged as kinematically anomalous extend the CTFR relation to very blue colors and large luminosity offsets, apparently reflecting interaction-induced star formation. Of course, kinematically anomalous galaxies probably experience luminosity boosts as well, and in fact two such galaxies are also exceptionally blue. However, anomalous galaxies are typically outliers from the CTFR relation, and their TF offsets may include velocity offsets. Unfortunately, it is presently impossible to separate luminosity and velocity offsets for these galaxies.

If, as expected from hierarchical merging scenarios, the galaxy interaction rate was higher in the past, kinematic anomalies may pose a serious problem for high- $z$ TF studies. Severe anomalies are roughly twice as common in the Close Pairs Survey as in the NFGS ( $\sim 20 \%$ vs. $\sim 10 \%$ of galaxies brighter than $M_{B}=-18$ ). Galaxy interactions probably explain the 3-4 times higher rate of strong rotation curve asymmetries in the Close Pairs Survey compared to the NFGS. The externally triggered gas inflow associated with interactions can also lead to centrally concentrated line emission and thereby rotation curve truncation, a problem that may be compounded by low $\mathrm{S} / \mathrm{N}$ data. However, our data are inconclusive as to the primary source of rotation curve truncation in the Close Pairs Survey. To the extent that gas inflow processes play a role at high $z$, some of the assumptions inherent in high- $z$ rotation-curve fitting techniques may break down, leading to artificially low velocity widths, as observed at low $z$.

The frequency of kinematic anomalies at high $z$ that would meet our criteria is presently unknown. We have shown that TF outliers associated with kinematic anomalies in the Close Pairs Survey occupy the same part of TF parameter space as the galaxies responsible for TF slope evolution in some high$z$ studies (e.g., Simard \& Pritchet 1998; Ziegler et al. 2002). These studies tend to have morphology-blind selection criteria that would include kinematically anomalous galaxies, which often have blue compact, emission-line S0, or peculiar morphologies. In contrast, studies that show less slope evolution (e.g., Vogt et al. 1997) tend to favor large disks and probably contain fewer kinematic anomalies.

We have also shown that the slope evolution in the high- $z$ FORS Deep Field sample (Böhm et al. 2003) is largely driven by "low-quality" data points, labeled as such by Böhm et al. based on perturbations or limited radial extent in the rotation curves. Using the FORS Deep Field data, we find that most or all of the TF evolution measured at high $z$ can be modeled as an overall $\sim 0.3$ mag luminosity offset at fixed slope, consistent with evolution along the CTFR relation, plus a differential evolution component associated with kinematically anomalous galaxies, which show offsets as large as $\sim 2$ mag at low luminosities but add only a small $\sim 0.2 \mathrm{mag}$ enhancement to the total TF offset for the survey. We note that the use of the outdated Pierce \& Tully (1992) TF calibration (superseded by Tully \& Pierce 2000) as a low- $z$ reference relation contributes $0.3-0.4$ mag of spurious luminosity evolution to many high- $z$ TF studies. At present, only the $\sim 0.3$ mag offset consistent with the CTFR relation can be reliably interpreted as luminosity evolution.
TF slope evolution associated with kinematic anomalies may be interesting for its own sake, as a source of data on massdependent evolution in the frequency of mergers and interactions (or the frequency of gas-dynamical disturbances caused by these events). Consistent with mass-dependent evolutionary trends in star formation histories and the luminosity function (e.g., Cowie et al. 1996), kinematically anomalous galaxies in the NFGS tend to be dwarf galaxies, fainter than $\mathrm{M}_{\mathrm{B}}=-18$, while analogous galaxies at high $z$ can be as bright as $\mathrm{M}_{\mathrm{B}} \sim-21$.

We have considered four strategies for isolating reliable luminosity offsets from offsets possibly associated with kinematic anomalies at high $z$ : identification of anomalies based on rotation curve properties, identification based on morphology, identification based on the color-TF residual relation, and inclusion of anomalies in optimally matched low- $z$ calibration samples that reproduce the distribution of anomalies expected at high $z$ (as well as high- $z$ selection criteria, data quality, and analysis techniques). The color-TF residual relation may offer the simplest and most powerful tool currently available for measuring luminosity evolution independent of kinematic anomalies at high $z$, especially when combined with optimal low-z calibration samples. Unreliable TF offsets associated with kinematic anomalies are typically CTFR outliers. Conversely, reliable luminosity enhancements lie on the CTFR relation and extend it toward bluer colors. Preliminary evidence for a CTFR relation at high $z$ has already been reported (Bershady et al. 1999). If the high $-z$ CTFR relation proves as tight as the Close Pairs Survey CTFR relation, then identifying CTFR outliers will serve as the preferred method for isolating kinematic anomalies in studies of luminosity evolution.

Once established, the high- $z$ CTFR relation may be applied to measuring not only luminosity evolution but also the evolution of stellar populations and stellar-to-total mass fractions (Kannappan et al. 2003a). The CTFR relation and the analogous relations for $\mathrm{EW}(\mathrm{H} \alpha)$ and $\mathrm{EW}([\mathrm{OII}])$ can also be used to reconcile discrepancies between high- $z$ TF studies with different selection biases in color or emission-line strength (Kannappan 2001; Kannappan et al. 2003a, 2004). Matching selection criteria at low and high $z$ is only the first step, however, because of the potential for luminosity-dependent evolution in the frequency of kinematic anomalies. By combining well-matched low- $z$ calibration samples with careful modeling of kinematic anomalies and the CTFR relation, future high- $z$ TF studies should be able to properly account for the major uncertainties of existing studies and reach consensus on how galaxy luminosities have evolved over cosmic time.

Data for the FORS Deep Field were kindly provided by A. Böhm, who also offered useful commentary on those data. We thank Niv Drory and David Koo for valuable feedback on this work, and Karl Gebhardt, Margaret Geller, and an anonymous referee for helpful comments on the manuscript. We also thank Dan Fabricant and Margaret Geller for the use of unpublished data from the NFGS and the Close Pairs Survey. EJB acknowledges support from NASA through Hubble Fellowship grant HST-HF-01135.01, awarded by STScI, which is operated by AURA for NASA under contract NAS 5-26555. This research has used NASA's Astrophysics Data System Bibliographic Services. 


\section{REFERENCES}

Böhm, A., Ziegler, B. L., Saglia, R. P., Bender, R., Fricke, K. J., Gabasch, A., Heidt, J., Mehlert, D., Noll, S., \& Seitz, S. 2003, astro-ph/0309263

Barton, E. J., Bromley, B. C., \& Geller, M. J. 1999, ApJ, 511, L25

Barton, E. J., Geller, M. J., Bromley, B. C., van Zee, L., \& Kenyon, S. J. 2001, AJ, 121, 625

Barton, E. J., Geller, M. J., \& Kenyon, S. J. 2000a, ApJ, 530, 660

Barton, E. J., Kannappan, S. J., Kurtz, M. J., \& Geller, M. J. 2000b, PASP, 112 , 367

Barton, E. J. \& van Zee, L. 2001, ApJ, 550, L35

Barton Gillespie, E., Geller, M. J., \& Kenyon, S. J. 2003, ApJ, 582, 668

Beauvais, C. \& Bothun, G. 1999, ApJS, 125, 99

Bell, E. F. \& de Jong, R. S. 2001, ApJ, 550, 212

Bershady, M. A., Haynes, M. P., Giovanelli, R., \& Andersen, D. R. 1999, in ASP Conf. Ser. 182: Galaxy Dynamics - A Rutgers Symposium, 499

Burstein, D. 2003, AJ, 126, 1849

Courteau, S. 1997, AJ, 114, 2402

Courteau, S. . \& Rix, H. 1999, ApJ, 513, 561

Courteau, S. \& Sohn, Y.-J. 2003, in The Mass of Galaxies at Low and High Redshift. Proceedings of the ESO Workshop held in Venice, Italy, 24-26 October 2001, 204

Cowie, L. L., Songaila, A., Hu, E. M., \& Cohen, J. G. 1996, AJ, 112, 839

Dale, D. A., Giovanelli, R., Haynes, M. P., Hardy, E., \& Campusano, L. E. 2001, AJ, 121, 1886

Forbes, D. A., Phillips, A. C., Koo, D. C., \& Illingworth, G. D. 1996, ApJ, 462, 89

Freeman, K. C. 1970, ApJ, 160, 811

Geller, M. J. \& Huchra, J. P. 1989, Science, 246, 897

Giraud, E. 1986, A\&A, 164, 17

Grogin, N. A. \& Geller, M. J. 1998, ApJ, 505, 506

Gunn, J. E. \& Gott, J. R. I. 1972, ApJ, 176, 1

Guzman, R., Koo, D. C., Faber, S. M., Illingworth, G. D., Takamiya, M., Kron, R. G., \& Bershady, M. A. 1996, ApJ, 460, L5

Haynes, M. P., Giovanelli, R., Chamaraux, P., da Costa, L. N., Freudling, W., Salzer, J. J., \& Wegner, G. 1999, AJ, 117, 2039

Heidt, J., Appenzeller, I., Gabasch, A., Jäger, K., Seitz, S., Bender, R., Böhm, A., Snigula, J., Fricke, K. J., Hopp, U., Kümmel, M., Möllenhoff, C., Szeifert, T., Ziegler, B., Drory, N., Mehlert, D., Moorwood, A., Nicklas, H., Noll, S., Saglia, R. P., Seifert, W., Stahl, O., Sutorius, E., \& Wagner, S. J. 2003, A\&A, 398, 49

Hernquist, L. \& Mihos, J. C. 1995, ApJ, 448, 41

Jansen, R. A., Fabricant, D., Franx, M., \& Caldwell, N. 2000a, ApJS, 126, 331

Jansen, R. A., Franx, M., Fabricant, D., \& Caldwell, N. 2000b, ApJS, 126, 271

Kannappan, S. J. 2001, PhD thesis, Harvard University

Kannappan, S. J. \& Fabricant, D. G. 2000, in ASP Conf. Ser. 230: Galaxy Disks and Disk Galaxies, eds. J. G. Funes \& E. M. Corsini, 449

Kannappan, S. J. \& Fabricant, D. G. 2001, AJ, 121, 140

Kannappan, S. J., Fabricant, D. G., \& Franx, M. 2002, AJ, 123, 2358

Kannappan, S. J., Fabricant, D. G., \& Franx, M. 2004, in preparation
Kannappan, S. J., Gillespie, E. B., Fabricant, D. G., Franx, M., \& Vogt, N. P. 2003a, in RevMexAA (SC) 17: Galaxy Evolution, theory and observations, eds. V. Avila-Reese, C. Firmani, C.S. Frenk \& C. Allen, 188

Kannappan, S. J., Jansen, R. A., \& Barton, E. J. 2003b, AJ, in press

Kobulnicky, H. A. \& Gebhardt, K. 2000, AJ, 119, 1608

Kornreich, D. A., Lovelace, R. V. E., \& Haynes, M. P. 2002, ApJ, 580, 705

Kraan-Korteweg, R. C., Sandage, A., \& Tammann, G. A. 1984, ApJ, 283, 24

Mallén-Ornelas, G., Lilly, S. J., Crampton, D., \& Schade, D. 1999, ApJ, 518, L83

McGaugh, S. S., Schombert, J. M., Bothun, G. D., \& de Blok, W. J. G. 2000, ApJ, 533, L99

Mihos, J. C. 2001, ApJ, 550, 94

Moore, B., Lake, G., \& Katz, N. 1998, ApJ, 495, 139

Mould, J., Han, M., \& Bothun, G. 1989, ApJ, 347, 112

Murali, C., Katz, N., Hernquist, L., Weinberg, D. H., \& Davé, R. 2002, ApJ, 571,1

Nilson, P. 1973, Uppsala general catalogue of galaxies (Uppsala: Astronomiska Observatorium, 1973)

Patton, D. R., Pritchet, C. J., Carlberg, R. G., Marzke, R. O., Yee, H. K. C., Hall, P. B., Lin, H., Morris, S. L., Sawicki, M., Shepherd, C. W., \& Wirth, G. D. 2002, ApJ, 565, 208

Pierce, M. J. \& Tully, R. B. 1988, ApJ, 330, 579

-. 1992, ApJ, 387, 47

Pisano, D. J., Kobulnicky, H. A., Guzmán, R., Gallego, J., \& Bershady, M. A. 2001, AJ, 122, 1194

Raychaudhury, S., von Braun, K., Bernstein, G. M., \& Guhathakurta, P. 1997, AJ, 113, 2046

Rix, H., Guhathakurta, P., Colless, M., \& Ing, K. 1997, MNRAS, 285, 779

Roberts, M. S. 1978, AJ, 83, 1026

Rubin, V. C., Burstein, D., Ford, W. K., \& Thonnard, N. 1985, ApJ, 289, 81

Rubin, V. C., Waterman, A. H., \& Kenney, J. D. P. 1999, AJ, 118, 236

Schlegel, D. J., Finkbeiner, D. P., \& Davis, M. 1998, ApJ, 500, 525

Simard, L. \& Pritchet, C. J. 1998, ApJ, 505, 96

- 1999, PASP, 111, 453

Sprayberry, D., Bernstein, G. M., Impey, C. D., \& Bothun, G. D. 1995, ApJ, 438, 72

Tully, R. B. \& Fisher, J. R. 1977, A\&A, 54, 661

Tully, R. B. \& Pierce, M. J. 2000, ApJ, 533, 744

Tully, R. B., Pierce, M. J., Huang, J., Saunders, W., Verheijen, M. A. W., \& Witchalls, P. L. 1998, AJ, 115, 2264

Verheijen, M. A. W. 2001, ApJ, 563, 694

Verheijen, M. A. W. \& Sancisi, R. 2001, A\&A, 370, 765

Vogt, N. P., Forbes, D. A., Phillips, A. C., Gronwall, C., Faber, S. M., Illingworth, G. D., \& Koo, D. C. 1996, ApJ, 465, L15

Vogt, N. P., Phillips, A. C., Faber, S. M., Gallego, J., Gronwall, C., Guzman, R., Illingworth, G. D., Koo, D. C., \& Lowenthal, J. D. 1997, ApJ, 479, L121

Willick, J. A. 1994, ApJS, 92, 1

Ziegler, B. L., Böhm, A., Fricke, K. J., Jäger, K., Nicklas, H., Bender, R., Drory, N., Gabasch, A., Saglia, R. P., Seitz, S., Heidt, J., Mehlert, D., Möllenhoff, C., Noll, S., \& Sutorius, E. 2002, ApJ, 564, L69 
FIG. 1.- Property distributions for the 73 NFGS and 88 Close Pairs galaxies used in our primary TF analysis (emission-line galaxies with $i>40$ and $\mathrm{M}_{\mathrm{B}}^{i}<-18$ ). The gray shaded and black cross-hatch histograms show the NFGS and Close Pairs Survey respectively. (a) B-band magnitudes. We have recomputed the Galactic extinction corrections for both surveys following Schlegel et al. (1998). Internal extinctions are computed based on Tully et al. (1998) as described in K02, except with no special treatment of S0 galaxies. (b) Morphological type. Numbers indicate a modified de Vaucoleurs type system, where $-1=\mathrm{S} 0,0=\mathrm{S} 0 / \mathrm{a}, 1-7=\mathrm{Sa}-\mathrm{Sd}, 8$ $=\mathrm{Sdm}, 9=\mathrm{Sm}$, and $10=$ both Magellanic irregular and unclassifiably peculiar galaxies. Classifications for the Close Pairs Survey were determined by Kannappan et al. (2003b) using the NFGS as a reference. (c) $B-R$ color within the half-light radius, corrected for Galactic and internal extinction. (d) Redshift in km s ${ }^{-1}$, corrected for Virgocentric infall and expressed relative to the Local Group. (e) B-band surface brightness at the half-light radius, with no profile decomposition. (f) Logarithm of the normalized environmental density. Densities are expressed in units of the mean density of galaxies brighter than $\mathrm{M}_{\mathrm{B}} \sim-17 \mathrm{smoothed}$ on $6.7 \mathrm{Mpc}$ scales, using code adapted from N. Grogin (Grogin \& Geller 1998). In these units the densities of the Virgo and Coma clusters are $\sim 4.9$ and $~ 7.4$ respectively, or $\sim 0.7$ and $\sim 0.9$ in logarithmic units. The mean density of 1 (logarithm $=0$ ) represents a field environment.
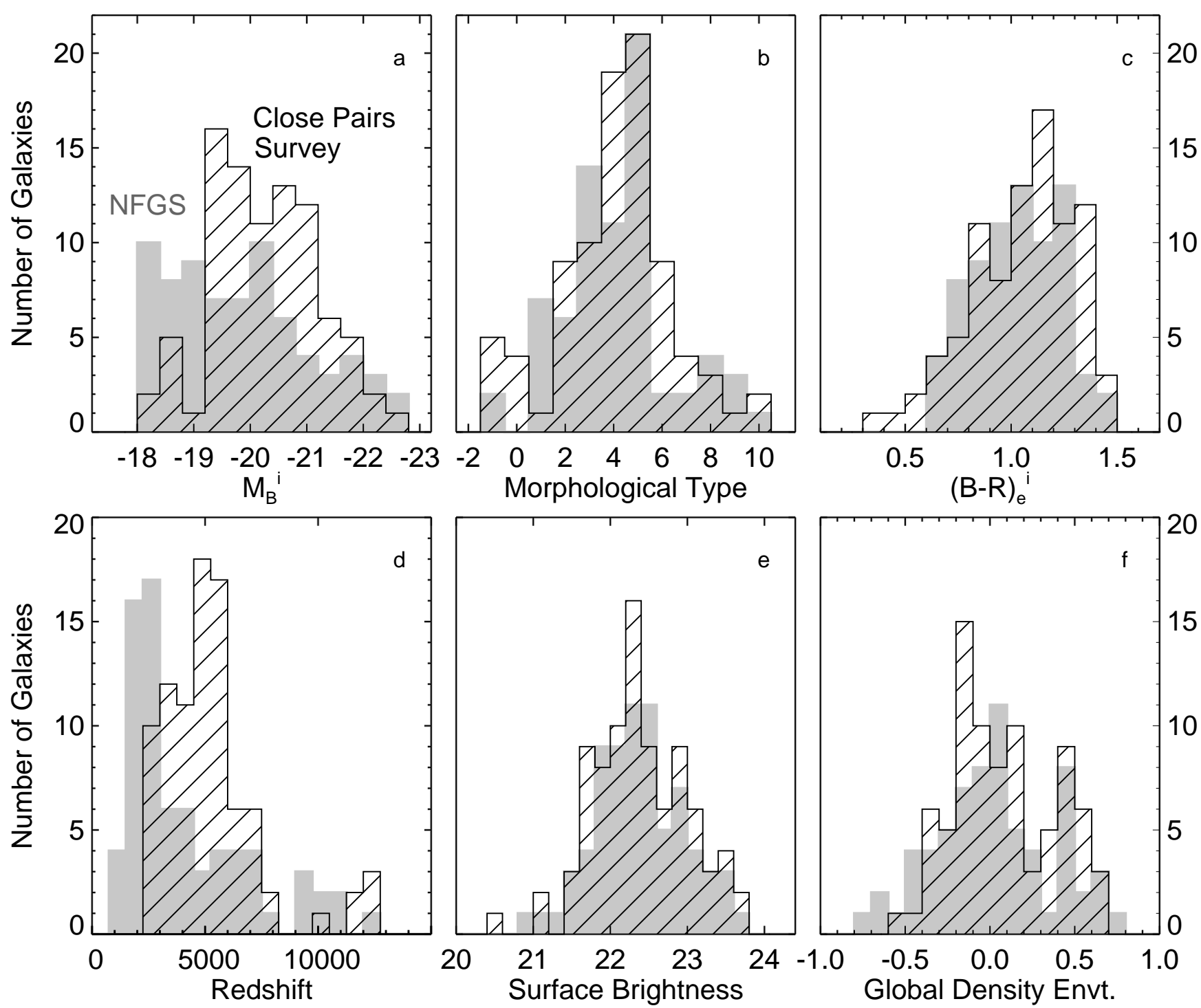



FIG. 2.- Rotation curves from the Close Pairs Survey. The first column and the top three examples in the second column are classified as kinematically anomalous (strongly asymmetric in shape and/or truncated in extent) by our quantitative criteria. The remaining examples do not meet these quantitative criteria, though a few show noticeable abnormalities. Letter identifiers correspond to labels used in $\S 4.1$. Measured asymmetries are noted in each panel. Dotted lines indicate $0.9 r_{e}$, the reference radius used to evaluate truncation. Dashed lines show the maximum and minimum velocities used to define $V_{p m m}$. Solid lines mark the coordinate center used for asymmetry calculations in each galaxy. We determine this center by minimizing the inner asymmetry (inside $1.3 r_{e}$ ) with the spatial center constrained to stay near the continuum peak and the velocity center allowed to vary freely. The final asymmetry is calculated over the full range of radii common to both sides of the rotation curve. For example, although the curve for galaxy M appears odd to the human eye, it shows only modest asymmetry $(6.5 \%)$ out to the largest radius at which flux is present on both sides of the galaxy. Truncation is measured from an average of the extent on both sides. 

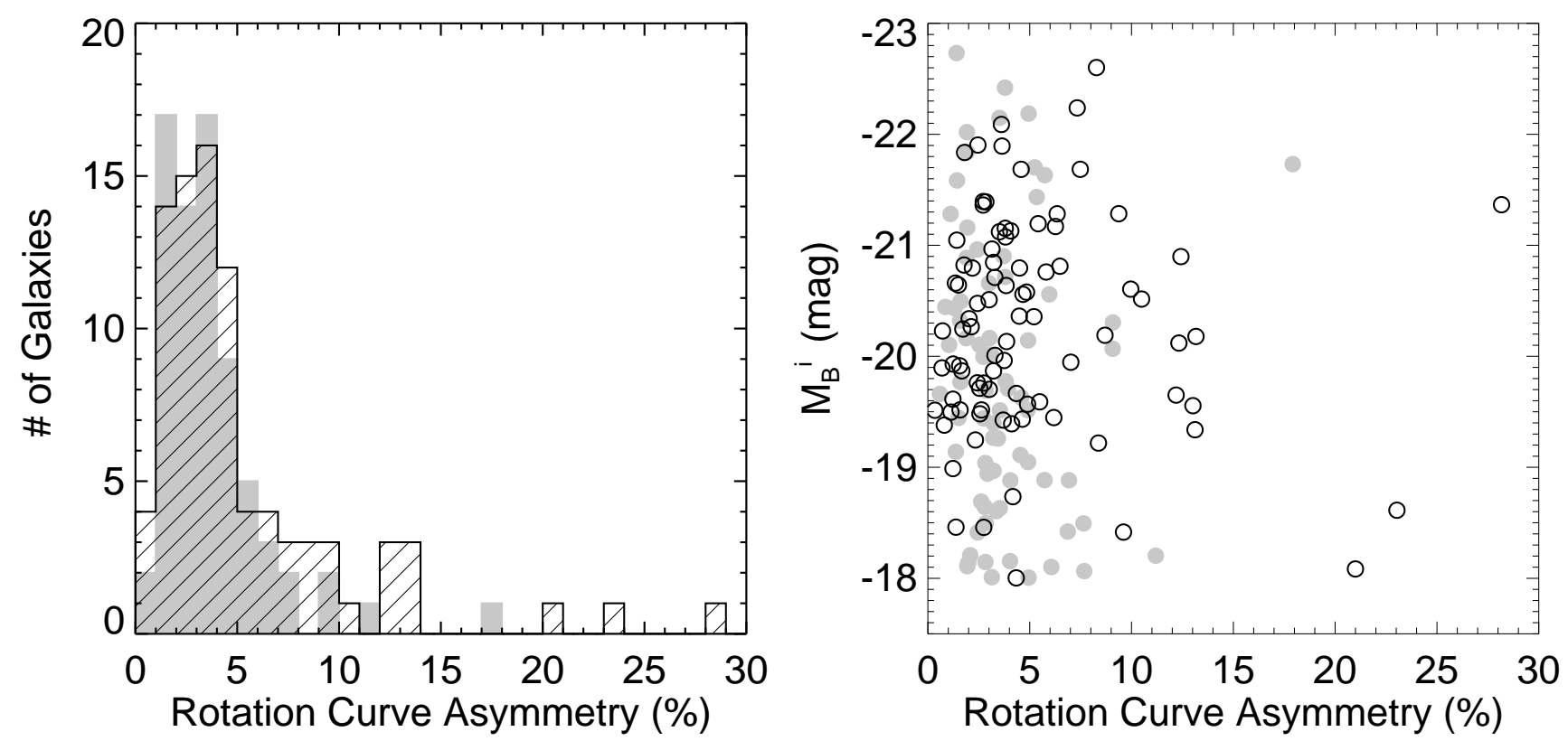

FIG. 3.- (a) Distribution of rotation curve asymmetries for TF sample galaxies in the Close Pairs Survey (black cross-hatched) and the NFGS (gray shaded). (b) Luminosity vs. rotation curve asymmetry for the same galaxies (Close Pairs Survey = open black circles; NFGS = gray dots).

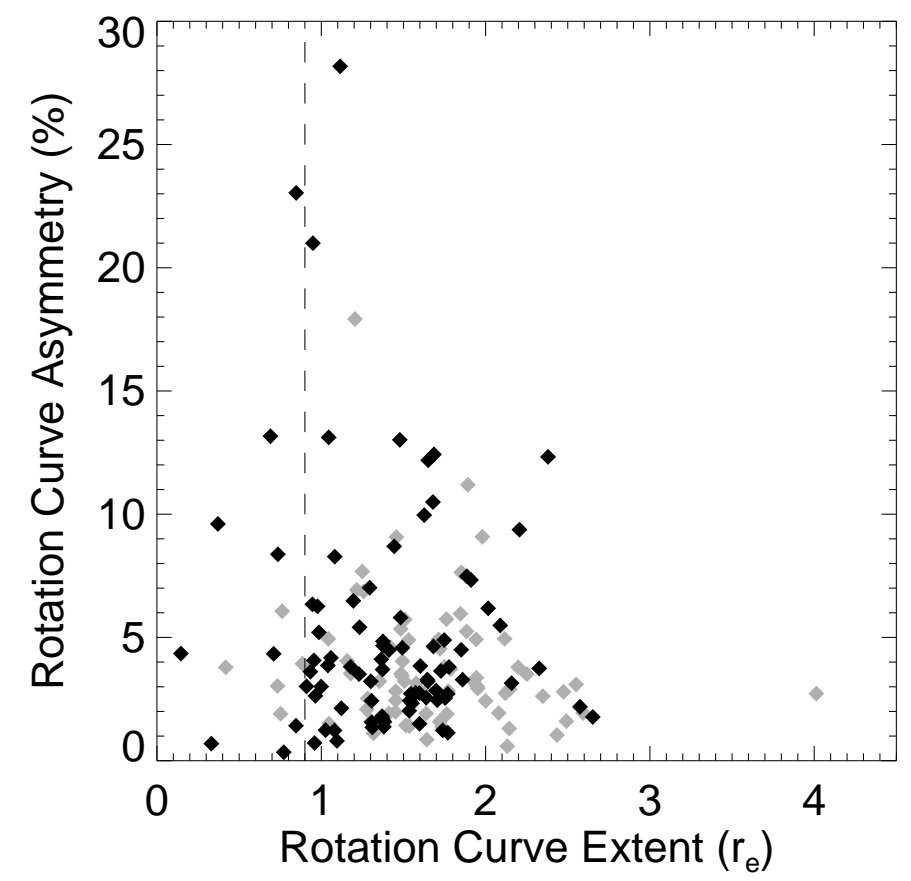

FIG. 4.- Rotation curve asymmetry vs. rotation curve extent for TF sample galaxies from the NFGS (gray) and the Close Pairs Survey (black). The vertical dashed line marks $0.9 r_{e}$. 

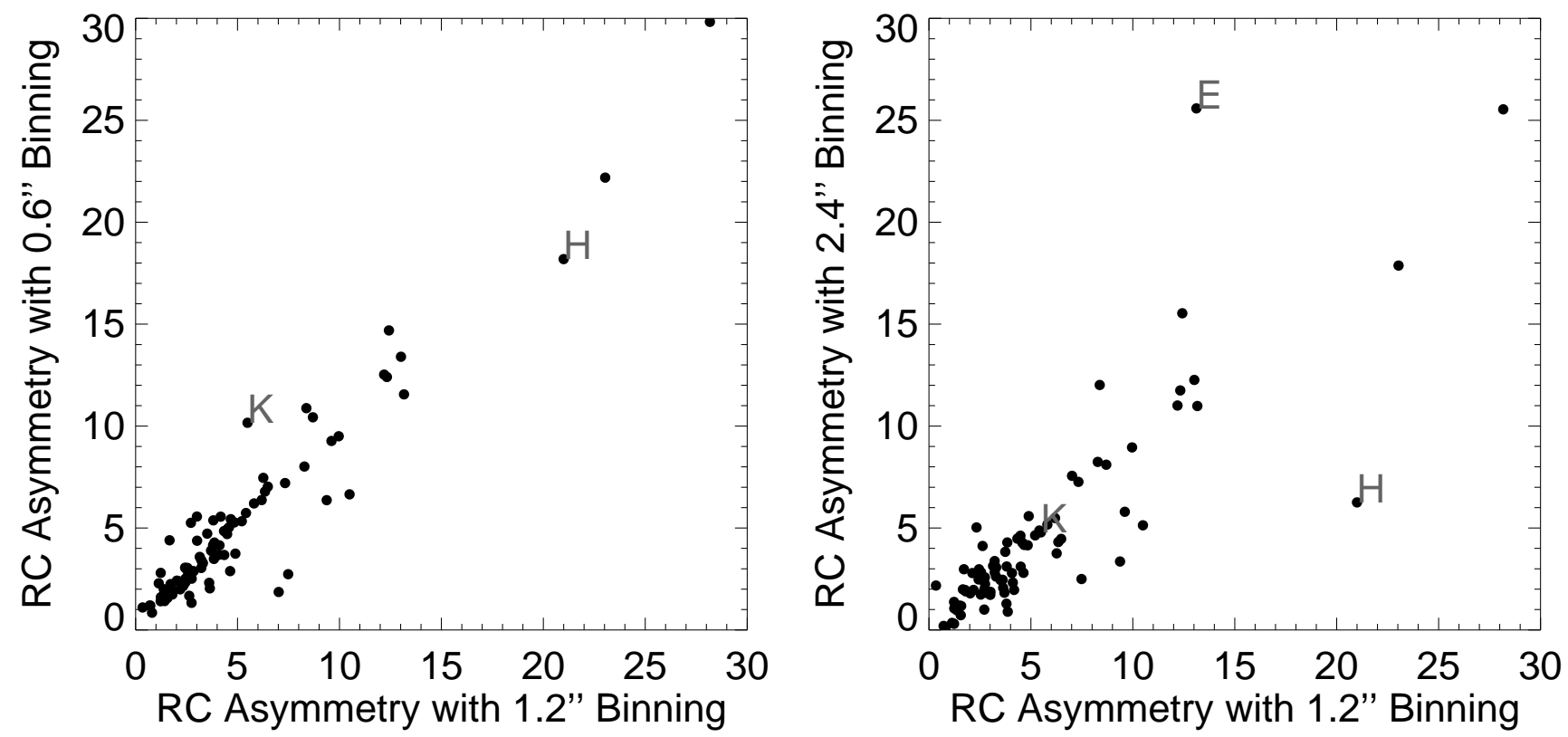

FIG. 5.- Comparison of rotation curve asymmetries measured with different spatial resolutions, using TF sample galaxies from the Close Pairs Survey. Letters refer to specific galaxies discussed in $\S 4.1$. Galaxy E does not appear in the left panel, as the lowest resolution data available for this galaxy have $1.2^{\prime \prime}$ binning. 

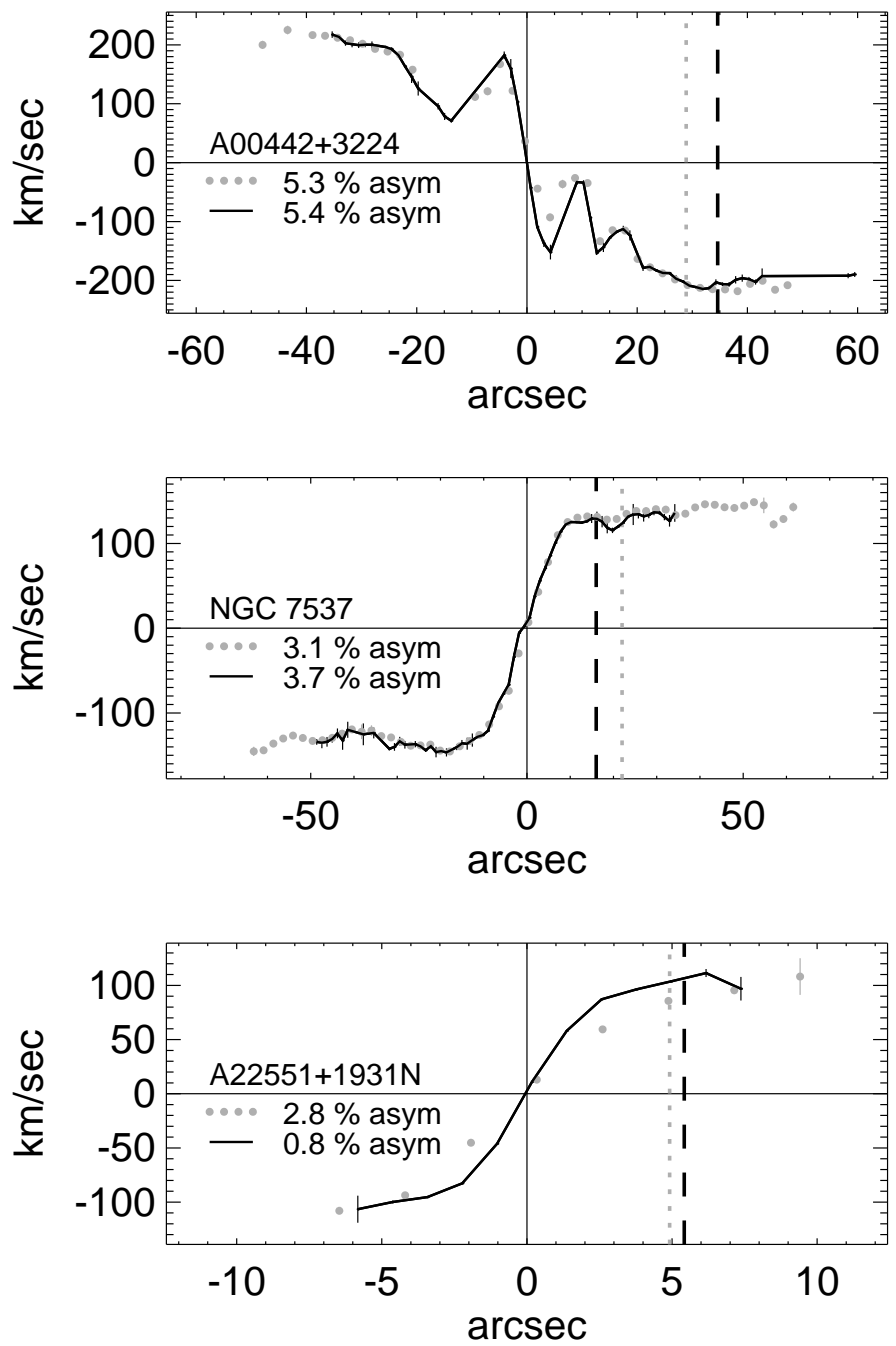

FIG. 6.- Rotation curves for the three galaxies common to both the NFGS (gray dots) and the Close Pairs Survey (black connected lines). Asymmetry measurements from the two surveys correlate well despite small differences in rotation curve structure caused by the lower spatial resolution of the NFGS and by different rotation curve extraction techniques (see note 5). Vertical lines indicate $0.9 r_{e}$, the cutoff radius used to identify truncated rotation curves $($ dashed $=$ Close Pairs Survey, dotted $=$ NFGS). These three galaxies all show adequate rotation curve extent for TF analysis. However, the variation between surveys illustrates the potential noisiness of our truncation measure. 

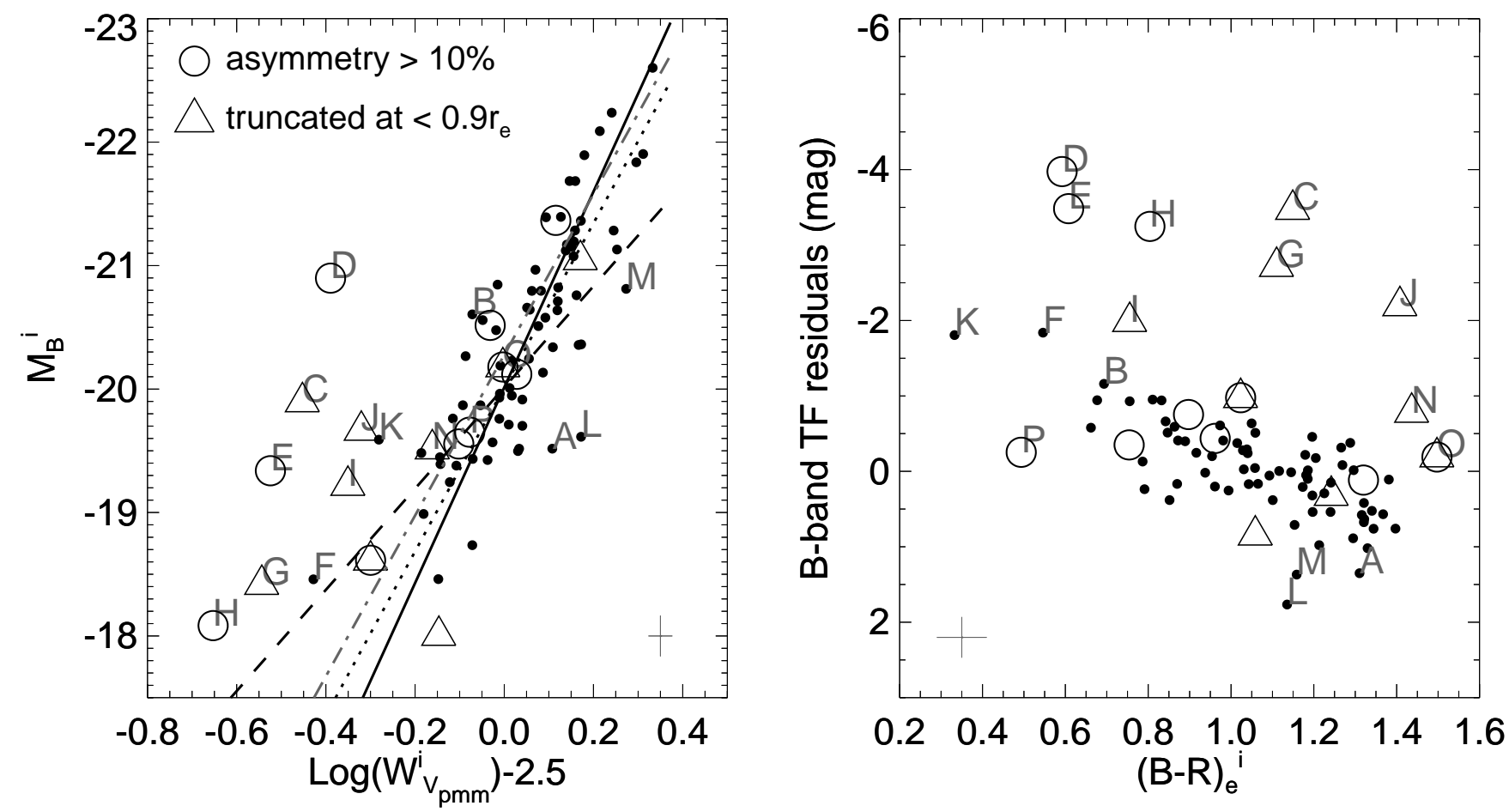

FIG. 7.- TF and CTFR relations for the Close Pairs Survey. Triangles and circles mark galaxies with truncated and asymmetric rotation curves, respectively; these galaxies are omitted from all TF fits except the dot-dashed line. The solid line shows the inverse-fit TF relation relative to which TF residuals for the CTFR relation are computed. The dotted line shows the bias-corrected forward-fit TF relation, shifted to the zero point of the inverse-fit relation. The dashed line indicates how much shallower the TF relation would need to be to make the CTFR relation statistically insignificant (see text). The dot-dashed line shows the inverse-fit TF relation obtained by restoring kinematically anomalous galaxies to the sample. Letters refer to specific galaxies discussed in the text, with letters A-H corresponding to the labels in B01. Crosses show representative error bars.
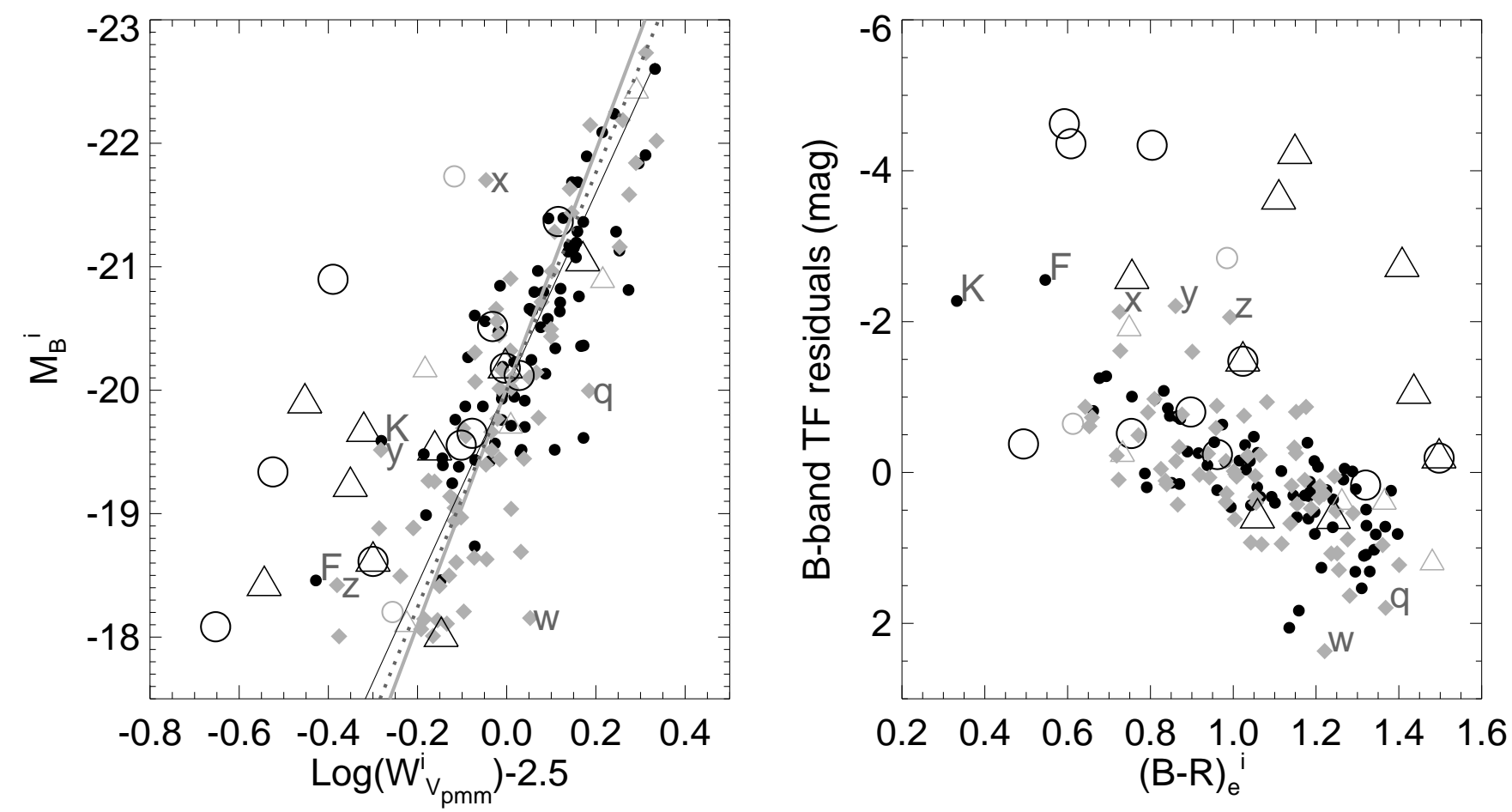

FIG. 8. - TF and CTFR relations for both the NFGS (gray diamonds, small gray circles and triangles, and solid gray line) and the Close Pairs Survey (black dots, large black circles and triangles, and solid black line). Triangles and circles mark galaxies with truncated and asymmetric rotation curves, respectively; these galaxies are omitted from all TF fits. The solid lines show inverse-fit TF relations for the two samples, and the dotted line shows the intermediate-slope relation obtained by either (a) omitting galaxies F and K from the Close Pairs Survey or (b) weighting the NFGS fit in proportion to the Close Pairs Survey luminosity distribution. We compute TF residuals for the CTFR relation relative to the solid gray line for both surveys. Letters indicate galaxies discussed in the text. 

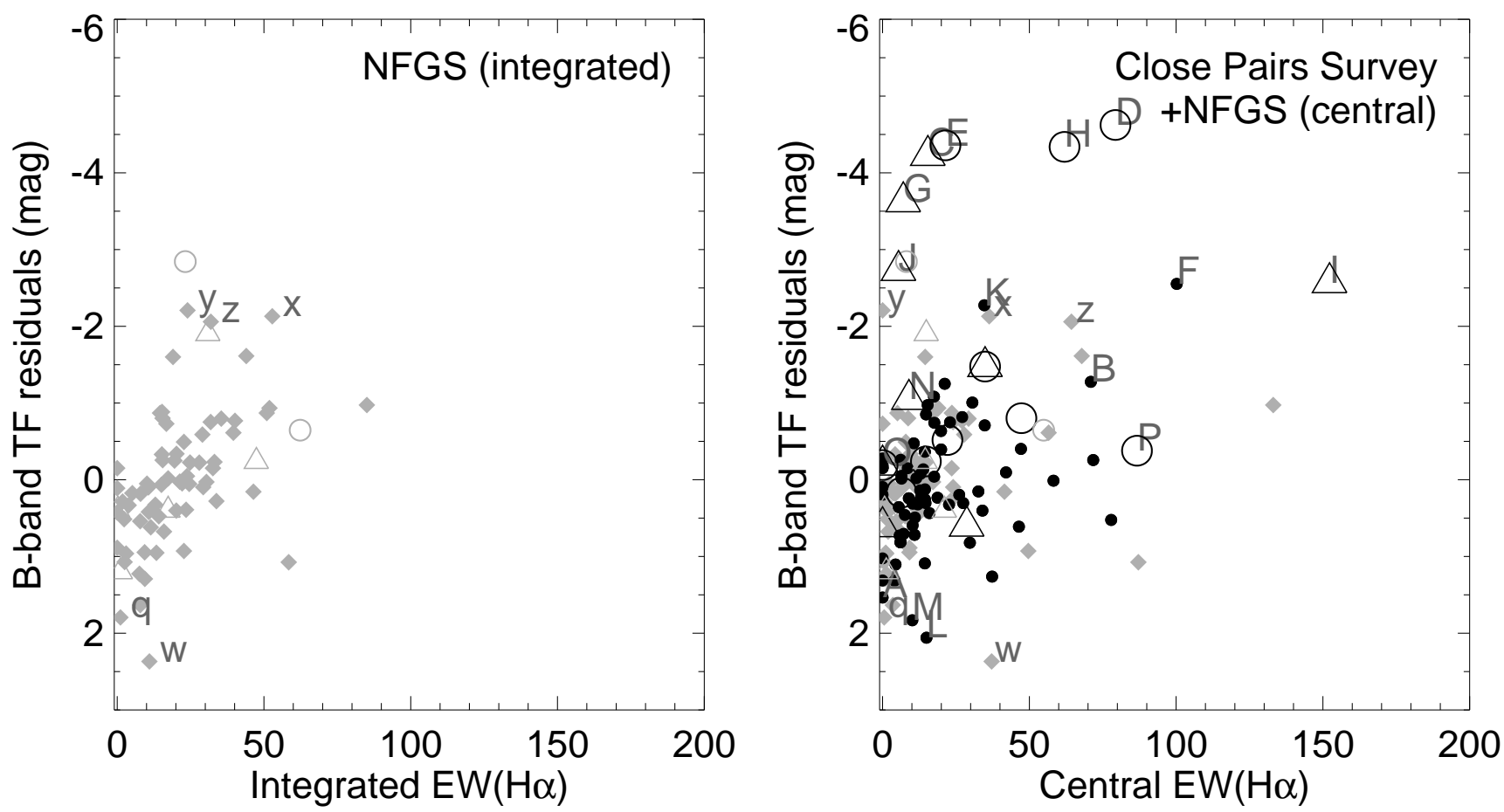

FIG. 9.- TF residuals vs. EW(H $\alpha$ ) for the NFGS (gray diamonds, small gray circles and triangles) and the Close Pairs Survey (black dots, large black circles and triangles). TF residuals are defined as in Fig. 8. Equivalent widths in the left panel are integrated over the entire galaxy (see Jansen et al. 2000a), while those in the right panel represent only a central aperture $\left(\sim 3^{\prime \prime} \times 7^{\prime \prime}\right.$ for the NFGS and $\sim 3^{\prime \prime} \times$ a variable length of $2-30^{\prime \prime}$ for the Close Pairs Survey). Integrated equivalent widths are not available for the Close Pairs Survey. Letters and symbols are as in Fig. 8.

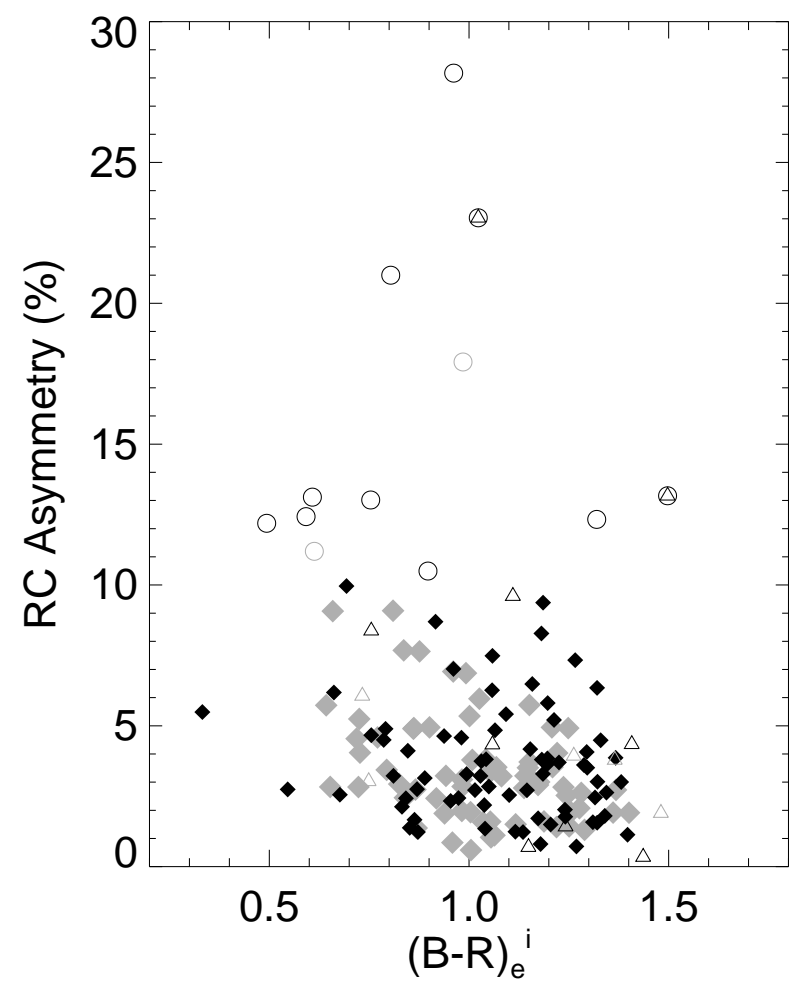

FIG. 10.- Rotation curve asymmetry vs. color. Gray symbols mark NFGS galaxies and black symbols mark Close Pairs galaxies (diamonds $=$ normal rotation curves; triangles $=$ truncated rotation curves; open circles $=$ asymmetric rotation curves). Spearman rank tests give $\sim 3.5 \sigma$ significance for the NFGS correlation and $\sim 2.5 \sigma$ significance for the Close Pairs correlation, using all galaxies shown. Excluding kinematically anomalous galaxies, the correlation strength drops to $3 \sigma$ for the NFGS, while the Close Pairs correlation is no longer statistically significant. 

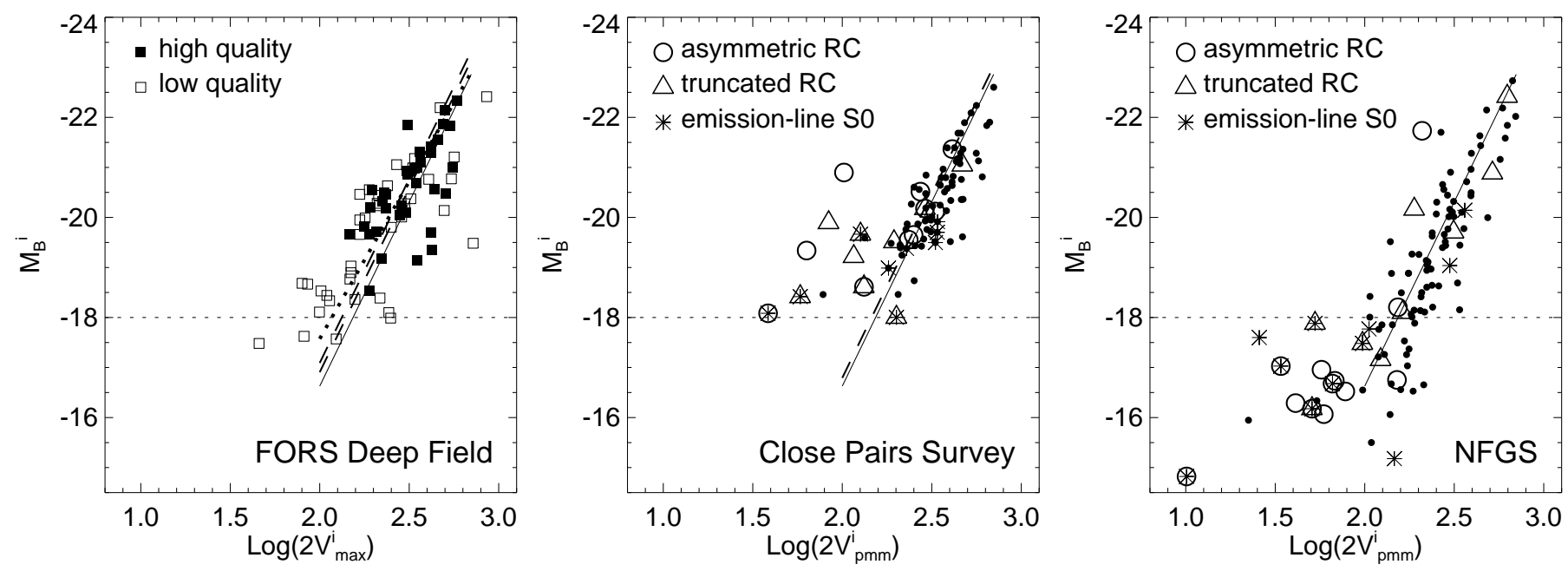

FIG. 11. - Tully-Fisher relations for the FORS Deep Field (general galaxy population at $z \sim 0.1-1$, data courtesy A. Böhm; Böhm et al. 2003), the Close Pairs Survey, and the NFGS (now including dwarf galaxies). Triangles and circles indicate severely truncated or asymmetric rotation curves in the two low- $z$ samples. Likewise, Böhm et al. flag "low-quality" data points in the high- $z$ sample based on limited radial extent or perturbations in the rotation curves. Data points are plotted as filled or open squares according to their high- or low-quality designations by Böhm et al. The solid line is a fit to the full NFGS Tully-Fisher sample, repeated in all three panels. Dashed lines indicate fixed-slope offset fits to the other two samples, with two lines to show fits with and without the low-quality data in the FORS Deep Field sample (larger and smaller offsets, respectively). The dotted line shows a free-slope inverse fit to the entire FORS Deep Field sample. Asterisks indicate emission-line S0 galaxies in the low- $z$ samples. We have converted the inclinations and internal extinction corrections for the high- $z$ data to our conventions, and shifted the data to our cosmology $\left(\mathrm{H}_{0}=75, \Omega_{m}=0.3, \Omega_{\Lambda}=0.7\right)$. 